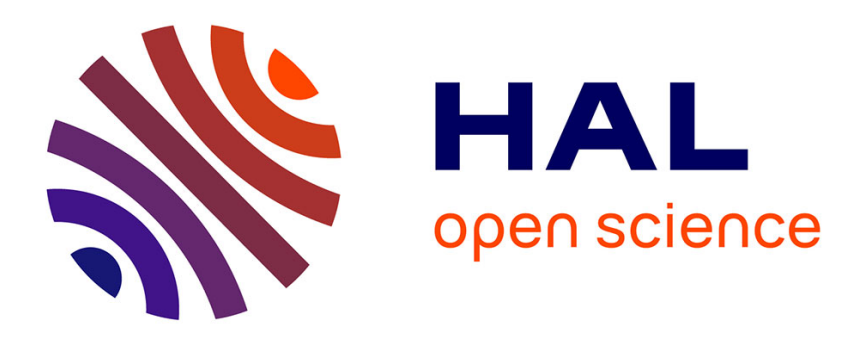

\title{
Mettre en perspective la perspective d'investissement social
}

\author{
Chantal Nicole-Drancourt
}

\section{To cite this version:}

Chantal Nicole-Drancourt. Mettre en perspective la perspective d'investissement social. La Revue de l'IRES, 2015, nº 85-86, pp. 171-209. 10.3917/rdli.085.0171 . halshs-01770960

\section{HAL Id: halshs-01770960 \\ https://shs.hal.science/halshs-01770960}

Submitted on 19 Apr 2018

HAL is a multi-disciplinary open access archive for the deposit and dissemination of scientific research documents, whether they are published or not. The documents may come from teaching and research institutions in France or abroad, or from public or private research centers.
L'archive ouverte pluridisciplinaire $\mathbf{H A L}$, est destinée au dépôt et à la diffusion de documents scientifiques de niveau recherche, publiés ou non, émanant des établissements d'enseignement et de recherche français ou étrangers, des laboratoires publics ou privés. 


\section{Mettre en perspective la perspective d'investissement social}

L'objet de cet article est de donner à voir la cohérence d'un chantier qu'on appelle communément la stratégie d'investissement social. Autour d'idées simples (concentrer les moyens sur la jeunesse et l'enfance) et de slogans entêtants (mieux vaut prévenir que guérir), cette stratégie affiche des objectifs partiels sans parvenir à valoriser ce que pourrait être le chantier une fois achevé. Cet article invitera donc à le faire à travers un questionnement spécifique : le degré de cohérence de la perspective d'investissement social dans ses référentiels d'action. Ce niveau de réalité sera saisi à travers les grands rapports prescripteurs mondiaux, régionaux et locaux et à travers la mise en forme des dispositifs de réformes en cours, notamment en France. L'argumentaire montre qu'aujourd'hui la perspective d'investissement social a fait son chemin, s'est frottée à l'épreuve des faits et s'est imposée au point de devenir une option sérieuse pour un imaginaire collectif alternatif.

La perspective d'investissement social ${ }^{2}$ est un puzzle complexe qui reste mal connu. Autour d'idées simples (concentrer des moyens sur la jeunesse et l'enfance pour sécuriser l'avenir), de slogans entêtants (« mieux

1. CNRS-Cnam-Lise (drancourtchantal@hotmail.com). Je tiens à remercier l'IRES pour ses remarques qui ont contribué à améliorer cet article et tout particulièrement Solveig Grimault, ses propositions et son soutien sans lesquels ce projet n'aurait pas abouti.

2. Nous rendrons compte souvent dans cet article de l'expression « investissement social » par son sigle «IS ». Nous parlerons de « perspective d'investissement social » (peIS) pour désigner le projet d'organisation sociale, économique, politique, philosophique dans son ensemble, le terme de " perspective " insistant sur le fait que ce projet n'a encore atteint nulle part son point d'horizon. Le terme simple d' investissement social » sera utilisé dans son sens commun pour rendre compte du contenu de différentes mesures qui se rattachent à ce projet d'IS. Le terme de "logique d'investissement social » sera utilisé pour désigner le registre d'action dans lequel s'inscrivent les mesures d'IS. Enfin, le terme d'« État d'investissement social » renverra à sa définition à savoir, l'affichage (par un pays où le rôle de l'État est fort) d'un régime d'État social qui renvoie au projet d'IS. 
vaut prévenir que guérir »), de projets controversés (l'activation ou l'individualisation de la protection sociale), le plaidoyer autour de cette perspective en a éclairé quelques zones sans parvenir à valoriser ce que pourrait être la cohérence et la valeur du chantier une fois achevé.

L'objet de cet article est de donner à voir la cohérence de ce chantier pour identifier le projet global dont il nous semble être porteur. Pour autant, attribuer aujourd'hui un contour précis à la perspective d'investissement social n'est pas simple. Pas simple si l'on veut traiter de l'investissement social d'un point de vue sociologique car très peu de sociologues français ont travaillé la question ${ }^{3}$. Pas simple ensuite parce que le caractère « en construction » de cette perspective dénie à quiconque d'en détenir la définition : l'investissement social est un «mot méthode » dont le signifiant chemine à l'épreuve des faits, sans aujourd'hui s'imposer ni sur la nature du chemin ni sur le contenu du concept à l'arrivée.

Cela dit, n'est-il pas temps de parler, au présent et institutionnellement, de l'investissement social ? Nous pensons que oui. D'une part parce que l'investissement social est une perspective ancienne dont le monde semble s'emparer à nouveau pour penser le $\mathrm{XXI}^{\mathrm{e}}$ siècle ; d'autre part parce qu'en France, le choix est fait aujourd'hui de "profiter de réformes en cours de la protection sociale pour compléter voire repenser les objectifs et les instruments des systèmes de protection sociale, en s'appuyant sur une nouvelle orientation générale, formulée en termes d'investissement social » (Palier, 2014). Cet article invitera donc à le faire à travers un questionnement spécifique : le degré de cohérence actuelle de la perspective d'investissement social dans les référentiels affichés institutionnellement. Dès lors, l'argumentation de cet article doit être lue à ce niveau de réalité et pour ce qu'elle est : un regard sur les " principes d'action » de la perspective et non une analyse critique des processus de mise en œuvre des réformes en cours.

Travailler la cohérence des référentiels de l'investissement social est négligé (voire nié) au profit de travaux critiques sur le grand chantier des réformes en cours. Cet article a donc pour objectif d'alimenter ce déficit, ce qui nous semble indispensable dans la mesure où, partout dans le monde, de nombreux choix de politiques économiques et/ou sociales ne sont aujourd'hui lisibles et compréhensibles que si on les situe dans une perspective d'investissement social.

Nous ferons donc l'hypothèse (et nous tenterons de démontrer) que la perspective d'investissement social (peIS) sert aujourd'hui de cadre majeur au chantier de construction d'un nouvel imaginaire social et démocratique ${ }^{4}$.

3. On trouve à ce jour quelques ouvrages pluridisciplinaires sur « pelS et protection sociale »: Guillemard (2008) ou, plus récemment, Gazier, Palier, Périvier (2014). Sinon, ce sont surtout les politistes ou les économistes qui s'expriment depuis deux décennies, notamment Bruno Palier ou Robert Salais, Jérôme Gautié et, bien sûr Jacques Delors.

4. Nous emprunterons la notion « d'imaginaire social » à Castoriadis : voir Chapel (2008). 
Nous nous appuierons pour cela sur des travaux scientifiques divers et sur le matériau d'enquête (peu utilisé en sociologie et pourtant très heuristique) que constitue les principaux textes et rapports internationaux qui ont ponctué, depuis le début des années 1990, l'élaboration progressive de cette nouvelle perspective. En fin d'article, nous illustrerons ensuite notre hypothèse par une entrée, celle des rapports de genre, et ce pour deux raisons : c'est une dimension que nous travaillons depuis de nombreuses années et, bien que discutée, nous défendons l'idée d'un fort potentiel émancipateur de la perspective de l'investissement social pour les femmes, dans certaines conditions de mise en œuvre.

\section{La perspective d'investissement social : un nouvel imaginaire social ?}

\section{I.1. La perspective d'investissement social : comment en parler?}

\section{Comment penser l'investissement social ?}

Comment penser l'investissement social, à partir de quoi, dans la mesure où cette perspective reste aujourd'hui un chantier qui, dans la plupart des cas, ne dit pas son nom ? Nous avons choisi de le faire dans un registre très particulier, celui dit des « univers sociaux légitimants ». Appelé « mise en ordre du social » chez Fraser ${ }^{5}$, « modes de reconnaissance disponibles » chez Butler (Jami, 2009), « principes de structuration des institutions d'intégration et de justice sociale » chez Rosanvallon (2006), « référentiels d'action » chez les analystes des politiques publiques ${ }^{6}$ ou « régimes » chez Esping-Andersen (1990), ce registre renvoie à un imaginaire d'organisation sociale, globale et historiquement située : là s'institue l'ordre des choses admises, là se réifient les légitimités, là sont répertoriées les manières d'agir attendues dans les relations sociales.

La sphère des « univers sociaux légitimants » est souvent rabattue sur de simples productions de l'esprit (en termes de visions du monde, de représentations, de préférences ou autres valeurs culturelles), ce qu'elle n'est pas. Cette sphère est une institution à part entière : elle constitue l'un des rouages essentiels de la grande "machinerie de construction du réel ${ }^{7}$ » car elle donne du sens aux échanges, interactions, actions et pratiques du quotidien. Cela dit, en rendre compte n'est pas simple car cette sphère n'est pas accessible à l'expérience. Sorte d'inconscient structurant de l'action, elle oblige le plus souvent à n'être saisie que dans ses manifestations. C'est

5. Registre des « modèles institutionnalisés de valeurs culturelles » (MIVC) : voir Fraser (2005).

6. Dans le sillage du néo-institutionnalisme et de l'institutionnalisme historique américain, l'école française cognitiviste s'intéresse aux univers de sens à travers l'analyse des cadres cognitif et normatif des politiques. Voir Jobert (1993) ; Faure, Pollet, Warin (1995) ; Muller (2005).

7. En s'appuyant sur les travaux issus de disciplines plurielles et en utilisant les outils analytiques à disposition, on peut imaginer styliser la « machinerie » de la dynamique de construction du réel. C'est ce que nous avons tenté dans un travail académique (Nicole-Drancourt, 2011). 
dans ces manifestations que nous irons donc principalement chercher et à ce titre, les grands rapports prescripteurs supranationaux sont des expressions majeures de la dynamique de construction de la perspective d'investissement social (peIS).

Saisir la peIS dans les manifestations de ses référentiels d'action relève d'une démarche constructiviste. La posture constructiviste, qui pousse à faire un pas de côté pour mettre au jour l'épaisseur du social, est particulièrement heuristique dans l'interprétation de la nature de certaines grandes mutations à l'œuvre aujourd'hui, notamment celles des transformations des politiques sociales et économiques.

\section{La posture constructiviste : un outil au service de l'analyse de la nature de la crise ?}

Cette posture permet notamment de s'écarter des analyses en terme de « crise » (déclin de l'État, déclin des institutions, déclin de la démocratie, déclin du lien social, etc.) au profit d'une autre conception de ce qui se joue aujourd'hui dans beaucoup de sociétés salariales contemporaines. Avec une posture constructiviste, on peut avancer qu'il s'agit moins de la crise d'un système due à son dysfonctionnement qu'à sa mutation due au processus de « décohérence » de son architecture ${ }^{8}$. La décohérence qui est questionnée ici est celle de l'inadéquation croissante entre deux registres : celui des cadres de reconnaissance et celui des pratiques sociales dans le «vivre ensemble ».

Les sociétés salariales de première modernité ${ }^{9}$ ont en effet longtemps présenté une adéquation étroite de leurs référentiels d'action (organisation fordiste de la production, mise en forme de la condition salariale, développement de l'État-providence, normes de conduite ; voir Castel, 1995) avec les pratiques sociales (modes de vie, parcours et comportements très standardisés) et la définition de leur environnement (richesses et développement de la planète infinis, maitrise de la nature, futur certain, etc.). Cette adéquation étroite chancelle dès la fin des années 1960 avec ce que l'on va progressivement définir comme « la montée des risques et de l'incertitude » (Beck, 1986/trad. fr. 2001) : ceux liés aux grands risques collectifs (environnementaux, climatiques), ceux liés au travail (fragilisation de la relation d'emploi, chômage, inemployabilité), ceux liés aux transformations de la famille (divorce et diversification des formes d'alliance) et à celle des relations sociales (baisse des grands collectifs d'action, forte aspiration à l'émancipation, à la reconnaissance et à la citoyenneté des «minorités identitaires et culturelles ", etc.).

8. En France, les travaux de François Ewald ou de Pierre Rosanvallon sur l'État-providence puis les scénarios alternatifs sur le travail présentés par Alain Supiot ou Jean Boissonnat sont représentatifs de cette posture en termes de mutation sociétale qui s'affirme à la fin des années 1990.

9. Pour reprendre les termes de Beck (1986/trad. fr. 2001) qualifiant toutes les sociétés industrialisées. 
Ces nouveaux risques ${ }^{10}$ génèrent, depuis près d'un demi-siècle, des réponses politiques, humaines et citoyennes au quotidien mais, si les fondamentaux des sociétés salariales de première modernité sont aussi l'objet de profondes remises en cause, ils restent, à ce jour, sans mode d'emploi alternatif légitime et encore moins universel. Le seuil de l'an 2000 peut alors être considéré comme le point d'orgue de la condition salariale traditionnelle et le point de départ d'un processus vers une seconde modernité à penser, à concevoir et à mettre en œuvre. Ce contexte invite donc à rebattre les cartes pour jeter les bases d'un «nouvel imaginaire » social ${ }^{11}$ et démocratique ${ }^{12}$.

\section{La perspective d'investissement social : un nouvel imaginaire démocratique des sociétés de seconde modernité ?}

On peut dire que cette invitation à « rebattre les cartes » a été comprise pour ce qu'elle était. Ainsi, à peu près partout dans le monde, les réformes en chantier depuis 20 ans dépassent la simple adaptation conjoncturelle pour poser la question du renouvellement structurel de l'organisation et du développement des sociétés. D’abord peu repérable au seuil de l'an 2000 mais de plus en plus lisible depuis, nous pensons qu'une alternative crédible commence à s'imposer autour d'une idée, celle de l'investissement social.

Cette notion insiste sur le fait que sans investissement social, l'investissement strictement économique n'arrivera pas à bout d'un développement durable. Mieux : que ce n'est plus l'investissement dans le capital « mort » qui est rentable à l'avenir mais celui dans le capital humain. Ces idées ne sont pas nouvelles en soi puisqu'elles sont nées dans les années 1930 en Suède avec les théories du «modèle social productif ${ }^{13}$ ». Cela dit, affaiblies par le chaos issu de la Seconde Guerre mondiale, noyées sous la vague de croissance issue de la reconstruction, minées par l'offensive ultra-libérale en faveur d'un « démantèlement total de l'État-providence » aux premiers signes de crise, elles vont se perdre dans l'oubli. Il faudra attendre le chaos issu de la période libérale pour que l'idée « investissement social » revienne en force comme une réponse crédible au renouvellement des fondements traditionnels du développement.

10. Ces risques sont caractérisés de " nouveaux " parce que, contrairement aux " anciens ", ils renvoient plus à la culture (le mode de vie des hommes) qu'à la nature : voir Giddens (1990/ trad. fr. 1994).

11. Au sens de Castoriadis, qui parle de « monde commun de significations pensées comme l'ensemble des mises en sens effectuées par l'imaginaire social, qui se déploie au sein des collectifs d'individus et à la seule condition duquel il peut y avoir individu et société ».

12. Imaginaire démocratique au sens de Tocqueville qui renvoie la démocratie à un mouvement de (re)construction perpétuel, autour d'imaginaires changeant vers un point fixe (le principe d'égalité).

13. Les théoriciens suédois du « modèle social productif » assuraient « qu'investir socialement » permettait de réduire les pertes et d'accroître les opportunités de développement : voir Morel (2013). 


\section{I.2. La perspective d'investissement social : la longue marche d'un mot méthode}

Mais pour autant, est-il justifié de parler de sens, de cohérence, d'ébauche de paradigme alternatif global quand on parle aujourd'hui de la peIS ? Nous pensons que oui si l'on garde à l'esprit que passer d'une idée portée par quelques voix (celle de «l'investissement social») à une perspective défendue à l'échelle mondiale (celle de la peIS) ne se décrète pas mais se construit par des faits qu'on peut observer et mesurer. C'est ce dont nous allons rendre compte, à un niveau partiel, dans les plis du réel, celui des énoncés axiologiques et/ou des grands principes. Nous proposons de suivre ainsi trois « moments » de cette construction : d'abord celui qui correspond au long "travail de la société sur elle-même » ${ }^{14}$; ensuite celui qui correspond au coup d'envoi de l'aventure; enfin celui qui correspond au processus de globalisation d'un langage commun.

\section{Moment 1 de la pelS : un long travail de la société sur elle-même}

L'élaboration du scénario peIS semble avoir puisé aux grandes dynamiques de mutations sociétales de la fin du $\mathrm{XX}^{\mathrm{e}}$ siècle et aux voix des instances réflexives qui en rendent compte dans l'espace public et dans l'espace académique.

Les grandes alertes sur les nouveaux risques et leur influence sur la conception du développement

À partir des années 1970, de nombreux collectifs (mouvements sociaux, luttes d'émancipation, collectifs d'experts et/ou d'intellectuels) alertent sur le coût humain du modèle de «modernisation » des pays développés et ses conséquences en termes de développement. Tous condamnent la dynamique de développement des pays industrialisés (trop économiste, trop dominatrice, trop industrialiste, trop prédatrice de matières premières, trop destructrice de l'environnement) et l'accusent d'accroître dangereusement, et partout dans le monde, la pauvreté et les inégalités (Giddens, 1990/ trad. fr. 1994) ; Beck, 1986/trad. fr. 2001). Parallèlement est dénoncé le coût humain du modèle d'aide au développement des pays pauvres qui consiste à mobiliser des apports externes pour les équiper et à exporter des solutions non concertées. Sous la pression des acteurs de ces espaces de contestations, de nouvelles approches s'imposent en matière de développement et deux dimensions de ruptures s'affirment : un développement moins industrialiste et plus participatif :

14. Le « travail de la société sur elle-même », au sens tourainien, qui parle ainsi de la capacité de la société d'agir sur elle-même et de se transformer par son « travail » qui renvoie alors tant à celui des acteurs de la connaissance (académiques, médiatiques, politiques, artistiques...) qu'à ceux des mouvements de lutte et d'émancipation. 
- un développement moins industrialiste : un consensus croissant s'affirme autour de la critique des schémas de développement passés. Dans cette critique, le capitalisme et « le dogme du pouvoir autorégulateur de marché » (i.e. le libéralisme) sont largement ciblés, mais pas seulement. Les modèles keynésiens, pourtant moins libéraux, sont aussi pointés du doigt : en mettant en place un régime providentiel qui permet l'écoulement d'une production de masse par la garantie et le maintien des revenus ${ }^{15}$, le keynésianisme fait dépendre le bien-être social de la rentabilité industrielle et, comme le libéralisme, met l'être humain au service de l'économie et la croissance aux commandes du modèle. Autrement dit, le keynésianisme comme le libéralisme s'attachent à une approche très " économiciste » du développement et à ce titre sont en proie à la critique ; critiques d'autant plus sévères que les potentialités à transformer la productivité en croissance (comme celle de transformer la croissance en création d'emplois) tendent à baisser depuis plusieurs décennies en dépit de la financiarisation croissante de l'économie (Joly, 1993). Ces alertes récurrentes sur les nouveaux risques associées à l'atonie des capacités de croissance et/ou à la dégradation des conditions de vie des sociétés développées, alimentent une aspiration croissante à rompre avec les modes de développement passés. La critique d'une vision industrialiste et économiciste du développement va alors de pair avec la valorisation d'une autre approche plus « écologique, plus globale et plus diversifiée » (Debonneuil, 2010) du développement.

Depuis les années 1970, s'esquisse alors dans les arènes discursives du monde entier, une approche alternative du développement dont les modes d'expression sont divers (volonté de transformation des indicateurs de richesses, proposition de nouveaux critères à prendre en compte dans le calcul des PIB nationaux, etc. ; voir Gadrey, Jany-Catrice, 2012). On retrouvera en Europe le cours de ce débat dans les discussions qui ont accompagné le Grenelle de l'environnement, cet ensemble de rencontres internationales organisées en France entre septembre et décembre 2007 (L'Écologiste, 2007). Dans ces arènes, la volonté d'un déplacement du centre de gravité des objectifs productifs des nations s'est fait sentir : il s'agit de mettre au cœur de la production tout ce qui contribue à améliorer " la machine humaine », qui devient dès lors l'enjeu premier de la productivité, de la croissance et de la compétitivité ;

- un développement plus participatif : si les grandes alertes sur les nouveaux risques ont influencé la conception du développement dans le sens d'un développement plus humain, elles ont aussi influencé sa conception dans un sens plus " participatif ». D'autant plus audible que l'échec des

15. Le keynésianisme (pour lequel la croissance, la production, l'emploi dépendent de la " demande ») préconise de soutenir la consommation par la mise en place d'un État social de type providentiel qui garantira le seuil de dépenses nécessaire au niveau de croissance souhaitée. 
politiques de développement des années 1980 et 1990 est patent, l'aspiration à la participation est issue de deux mouvements démocratiques : d'une part, les mobilisations autour des «théories de la dépendance » qui, en dénonçant l'objectif visé (le pillage des ressources des pays dits aidés), lèvent le voile sur l'absurdité de la méthode (exporter des modèles de croissance de pays riches vers des pays pauvres); d'autre part, la demande de reconnaissance des savoir-faire et capacités citoyennes pour une résolution endogène des difficultés fait son chemin. Les grands organismes mondiaux (Pnud ${ }^{16}$, Unesco ${ }^{17} \ldots$... s'emparent alors de ces critiques pour afficher, dès la fin des années 1980, «le droit de participer, de contribuer et de profiter du développement économique, social, culturel et politique de son pays comme un droit inaliénable ${ }^{18} \%$. Les systèmes de communication nouveaux et alternatifs seront alors considérés comme des moyens importants de mise en œuvre de l'aide, pensée dorénavant en termes de co-construction, avec la participation des communautés locales à la définition des objectifs, à la formulation des politiques et à la mise en œuvre des programmes de développement (Jones, 2006).

Les grandes alertes sur les nouveaux risques ont donc joué un rôle majeur pour replacer la croissance au service d'un développement plus humain et plus participatif. Nous verrons que la logique d'action et d'intervention sociale issue de la perspective d'investissement social puisera largement à ces idéaux.

\section{La reconnaissance de l'expertise académique et son influence sur la conception du développement}

- La reconnaissance des recherches sur les nouveaux principes de justice : dans les « régimes d'État-providence ${ }^{19}$ ", la logique qui sous-tend la protection sociale est dite « ressourciste». Adossée à la fois sur une conception des droits naturels dans le contrat social ${ }^{20}$, sur les théories de la croissance de Keynes et sur les théories de la justice de Rawls, cette approche consiste à « égaliser les chances » en mettant à disposition des « ressources 》 (sous forme d'équipements publics ou sous forme de pouvoir d'achat ; Boyer, 2015) à tout ayant-droit. La reproduction des conditions sociales et les inégalités persistantes ont donné lieu à des nombreuses critiques de cette approche mais c'est un courant de pensée (porté par l'économiste

16. Programme des Nations unies pour le développement.

17. United Nations Educational, Scientific and Cultural Organization.

18. Voir la "Déclaration sur le droit au développement ", adoptée par l'Assemblée générale des Nations unies le 4 décembre 1986. Dans ce document, on ne parle plus de charité mais d'autonomisation et de participation en mettant les gens au centre du processus de développement.

19. Voir la notion de régimes (REP) chez Gøsta Esping-Andersen, figure stylisée qui résume l'ensemble des dimensions qui président, dans chaque pays, aux ordres/arrangements/conventions/ contrats sociaux et à la répartition des ressources dans les différents États-providence.

20. Postulat qui s'enracine dans les théories du contrat social : ignorant le caractère social et acquis des compétences humaines, le biologique est considéré comme le noyau originel et naturel à partir duquel doit se composer la vie sociale. À partir de là, les individus sont considérés comme différents mais naturellement égaux, libres et compétitifs (Nicole-Drancourt, 2011, op. cit.). 
Amartya Sen) qui symbolisera la rupture vers une conception nouvelle des référentiels d'action en matière de justice sociale. Prouvant par ses travaux qu'une même ressource affectée à des ayant-droit non semblables n'a pas les mêmes effets, Sen montre l'importance de la prise en compte des rapports sociaux qui placent les individus dans des positions inégalitaires qui les privent de liberté d'action. Sen défendra alors l'idée que les ressources externes n'apportent rien si l'on oublie la capacité réelle des individus à s'en emparer. Ce qu'il nomme «capacité » est ici une catégorie de la pensée, un outil pour l'action : pour que les individus transforment les opportunités en ressources réelles (processus qu'il nomme "functioning »), il faut leur garantir des droits « capacités » (instrument qu'il nomme « capabilities») qui permettent aux individus d'exercer « une liberté de choix réelle d'accomplir le projet de vie qu'ils ont des raisons sociales à valoriser» (Sen, 1979).

Cette conception (dite «non welfariste») s'éloigne des valeurs solidaristes traditionnelles : elle rompt avec les catégories «d'incapacité » ou « d'assisté » pour leur préférer celle « d'autonomie » ou « d'acteur responsable » (Sen, 1991) (que le sens commun en Europe renvoie à la seule responsabilité individuelle). Dès lors ces nouvelles théories de la justice seront beaucoup interprétées, souvent critiquées, mais elles inspireront néanmoins des économistes, des sociologues et des politistes de la planète entière (de Munck, Zimmermann, 2008) ${ }^{21}$. Parallèlement, l'ONU, la Commission des droits de l'homme et l'OCDE reprendront à leur compte une bonne partie (sinon l'ensemble) de la grammaire d'Amartya Sen pour repenser la dynamique de lutte contre la pauvreté : ainsi la question de l'autonomisation des individus, qui puise directement à celle de capacitation dans la définition, dans les mises en forme et dans la mise en œuvre des politiques contemporaines de développement. Nous verrons que la perspective d'investissement social puisera elle aussi largement à cette approche non welfariste de la justice sociale.

- La reconnaissance des recherches sur le capital humain et ses prolongements vers les théories de la croissance endogène : la notion de capital humain rassemble l'ensemble des savoir-faire et des savoirs utiles (actifs ou potentiels) chez un individu. Très peu mobilisé par le capitalisme de la première révolution industrielle, le capital humain disponible est devenu un enjeu majeur à la fin de la seconde révolution industrielle, lors de la première vague d'automatisation de la production. Aujourd'hui, au seuil de la troisième révolution industrielle, la question du capital humain (tant par sa qualité que par sa quantité) est pensée comme une condition majeure de son succès. L'économiste Gary Becker, désigné comme le père fondateur de cette théorie (Becker, 1964) ${ }^{22}$, prouve alors qu'un lien existe entre l'investissement d'un individu en éducation/formation et sa rémunération

21. Sen recevra aussi le prix Nobel d'économie en 1998 .

22. Et consacré comme tel par le prix Nobel d'économie en 1992. 
future. Mais cela suffit-il à expliquer l'engouement récent et unanime des acteurs et des décideurs de la planète pour ses théories ? Peut-être pas. De notre point de vue, tout se passe comme si cet engouement s'expliquait plutôt par l'intérêt croissant des décideurs pour d'autres théories qui empruntent au capital humain pensé par Becker (certainement), mais pour mieux s'en détacher peut-être et l'enrichir (sûrement).

En effet, que l'investissement dans le capital humain soit « rentable » pour une personne et que cette personne ait intérêt à s'en charger individuellement était compris depuis longtemps. Mais que l'investissement dans le capital humain soit « rentable » pour l'économie et qu'une société ait intérêt à s'en charger collectivement restait à prouver. C'est ce qu'ont tenté de faire les économistes des nouvelles théories de la croissance en général et celles de la « croissance endogène » en particulier (Guellec, Ralle, 2003) en mêlant dans un cercle vertueux « investissement dans le capital humain », " créativité », « optimisation du capital physique » et, surtout, croissance ${ }^{23}$.

Ce faisant, ces économistes ont su répondre à une inquiétude majeure : comment retrouver une croissance durable, résiliente aux nouveaux risques? Tout en affirmant que les taux de croissance passés sont à jamais compromis, les partisans de la croissance dite « endogène » maintiennent l'espoir en promettant qu'une autre croissance (plus soutenable, plus durable) est possible si l'on en change le logiciel. Dans ce cadre, ils proposent de mettre au cœur du processus de croissance l'investissement, compris comme le produit « d'acteurs agissants » dans quatre espaces principaux (Guellec, Ralle, 2003) : les rendements d'échelle (certains investissements peuvent entraîner des rendements croissants qui augmentent le capital physique et poussent la croissance); la recherche/innovation (réintégrée au cœur du modèle, l'innovation préside aux facteurs de production traditionnels) ; le capital humain (redéfini dans son contenu avec la formation, les compétences et la santé) et l'action publique (redéfini dans son rôle qui s'élargit au financement et la circulation des biens, des personnes et de l'information ${ }^{24}$ ). Autrement dit, ces théories considèrent que le savoir, la connaissance, la santé ou le social sont des investissements « rentables », et que l'État-redistributeur peut être aussi un État-investisseur.

On remarque à nouveau que s'éloignent des valeurs solidaristes traditionnelles : le social peut-il être "rentable » au même sens qu'un capital fixe ? Les personnes seraient-elles devenues de la "matière première", des marchandises, au même titre que le pétrole ? Cela n'a pas manqué de

23. Le premier modèle de croissance endogène a été publié par Paul Romer en 1986, puis le modèle a été principalement développé par Robert E. Lucas et Robert Barro. Pour une présentation d'ensemble, voir Guellec, Ralle (2003, op. cit.).

24. Quand « la productivité sociale d'une infrastructure est supérieure à la productivité privée d'une dépense », l'État doit financer. 
faire débat et celui-ci est loin d'être clos ${ }^{25}$. Cela dit, ces thèses sur le capital humain et la croissance endogène n'en inspirent pas moins de grandes ambitions. Ainsi, depuis 30 ans, on les retrouve dans tous les grands textes supranationaux prescripteurs : le Pnud reprend la notion de capital humain pour penser le développement dit humain ; l'OCDE propose régulièrement des rapports portant sur « capital humain et nouvelle croissance » et toutes les feuilles de route se réclamant d'une croissance durable s'appuient aujourd'hui sur des piliers de réformes où le développement du capital humain est un des éléments majeurs.

Au-delà des controverses, l'émergence du scénario d'investissement social puisera à toutes ces grandes dynamiques : les grandes alertes sur les nouveaux risques vont alimenter une volonté affichée à vivre, produire, consommer, travailler, participer « autrement »; les nouveaux principes de justice vers plus de responsabilité et d'autonomie vont rencontrer l'aspiration croissante aux émancipations et libertés citoyennes; et les audaces prospectives des économistes «mainstream » de la croissance endogène et du capital humain vont séduire les plus libéraux... mais aussi les plus solidaires qui s'appuient sur la critique pour fertiliser les concepts. Au titre d'utopie réalisable, et sans le proclamer comme tel, la perspective d'investissement social serait-elle en campagne pour servir de cadre au chantier de construction d'un « nouvel imaginaire social et démocratique » ?

\section{Moment 2 de la pelS : Rio 1992 ou le coup d'envoi de l'aventure}

La perspective d'investissement social serait-elle un «nouvel imaginaire social et démocratique »?

Pour justifier qu'on puisse répondre oui à cette question, nous proposons d'en dater l'acte d'officialisation : Rio 1992. On peut en effet interpréter la Conférence internationale sur l'environnement et le développement (que l'Onu organise à Rio du 3 au 14 juin 1992) comme le point d'orgue du long travail collectif de remise en cause des modèles de développement passés dont nous venons de parler. Avec le label « Premier Sommet de la Terre », l'événement ne cache d'ailleurs pas son ambition. Avec son objectif également, puisque l'enjeu de la conférence est la ratification par 173 chefs d'État du «Plan d'action pour le XXI ${ }^{e}$ siècle » (dit Agenda 21 ou Action $21^{26}$ ), programme qui se compose d'une déclaration (énonçant 27 principes), d'un programme d'action en 40 chapitres (et environ 2500 recommandations) et d'une feuille de route (la traduction progressive des principes et du programme dans les politiques publiques et les initiatives des acteurs économiques, sociaux, associatifs du monde entier).

25. Pour les premiers regards critiques chez les économistes, voir Fougeyrollas, Le Mouël, Zagamé (2005).

26. http://www.un.org/french/ga/special/sids/agenda21/action0.htm. 


\section{Le rapport Brundtland et le premier rapport mondial du Pnud : textes fondateurs de l'investissement social ?}

\section{Rapport Brundtland (1987). Notre avenir à tous (Our Common Future)}

Le rapport Brundtland, c'est l'invention de la notion de « développement durable ». Rédigée en 1987 par la Commission mondiale sur l'environnement et le développement de l'Onu présidée par la Norvégienne Gro Harlem Brundtland, le rapport utilise pour la première fois l'expression de " sustainable development », traduit en français par « développement durable », et il lui donne une définition : "Le développement durable est un développement qui répond aux besoins du présent sans compromettre la capacité des générations futures de répondre aux leurs ». Le rapport est un appel en direction du monde pour définir un programme de coopération internationale et pluridisciplinaire sur les problèmes environnementaux. Inhérentes à cette notion de développement durable, plusieurs notions sont mises en avant pour la première fois comme celles de "capacités ", de "bien premier " ou de "droits humains ». Depuis ce rapport, et dans une pluralité d'approches qui n'a d'égale que la diversité des mouvements qui s'en réclament, l'expression « développement durable » s'est répandue dans le monde entier.

\section{Rapport Pnud (1990). Rapport mondial sur le développement humain 1990}

Premier des rapports annuels du Pnud, le Rapport mondial sur le développement humain affiche l'objectif de replacer les personnes au centre du processus du développement, du point de vue du débat économique, des orientations de politique publique et de la sensibilisation : il s'agit d'entraîner le développement des personnes, par les personnes et pour les personnes, les buts du développement étant les choix et les libertés. Trois grandes ruptures s'affichent pour la première fois dans ce rapport :

- faire évoluer l'appréhension du concept de développement. Traditionnellement entendues comme phénomènes externes, apportées " d'en haut » par les sociétés occidentales modernes et financées sans concertation par la Banque mondiale, les notions de développement et de progrès sont présentées radicalement autrement dans ce rapport. En passant de l'idée de développement strictement " économique " au développement dit " humain ", le Pnud élargit la notion de développement à celles de liberté politique, économique ou sociale, à celle de créativité, à celle de respect de soi et à la garantie des droits humains fondamentaux. En contrepartie, l'humanité est appelée à s'orienter vers un développement « durable » : reconnaître aux hommes le droit au développement inclut le devoir de le mener d'une manière telle qu'il ne compromette pas le futur de la planète. Le rapport traite aussi de la signification et de la mesure de ces nouvelles conceptions du développement en proposant un nouvel indicateur composite ;

- faire évoluer l'appréhension du concept de pauvreté. L'évaluation du développement par le Pnud ne se fera plus en fonction d'indices purement 
économiques (comme le revenu par habitant ou le produit intérieur brut) mais aussi en fonction d'indicateurs en matière de santé ou d'éducation. II s'agira alors de qualifier la pauvreté en termes d'obstacle à l'exercice des droits de l'homme et de tenir compte de sa nature multidimensionnelle : elle n'est plus à considérer comme une simple pénurie de revenus économiques mais comme la privation de "capacités " (" capabilities » au sens d'Amartya Sen).

- rompre avec l'idée de croissance et privilégier celle d'investissement. En ne parlant plus de développement mais de développement humain et durable, la croissance est considérée comme nécessaire mais non suffisante pour assurer le développement humain. C'est une prise de conscience, celle qu'en dépit d'une croissance rapide du PNB ou d'un revenu par habitant élevé, le progrès peut faire défaut. Avec le slogan « le développement humain est une fin dont la croissance économique est le moyen ", le Pnud reprend l'idée chère aux théories du capital humain en l'inversant : l'accumulation de capital humain permet des gains de productivité favorables à la croissance et à l'emploi, mais les hommes sont plus que des moyens (machines à fabriquer) au service d'une fin (les biens de consommation). Agents du développement, les êtres humains sont considérés ici comme bénéficiaires et fin ultime du processus. II faut donc investir dans le développement humain pour que les individus soient et parce qu'ils sont la véritable richesse d'une nation.

Pour faire encore plus le lien entre la feuille de route présentée à Rio, le long travail qui l'a précédée et la perspective « d'investissement social », il faut prendre connaissance des deux rapports (le rapport Brundtland et le premier rapport mondial du Pnud) qui ont servi de textes institutionnels fondateurs à l'élaboration du Programme Agenda 21 (encadré 1).

Dans cette présentation rapide des deux rapports, il est très clair que leur contenu reprend largement le chantier de réflexion à l'œuvre dans les arènes discursives sur l'avenir du monde en matière de développement, de participation et de croissance (Deacon, 2001). En fait, nul « auteur » ne s'exprime dans le rapport Brundtland ou dans celui du Pnud : les ruptures qui s'affichent dans le rapport du Pnud puisent directement aux grandes alertes et aux mobilisations de la société civile. Le rapport Brundtland et le rapport $\mathrm{n}^{\circ} 1 \mathrm{du}$ Pnud ne sont donc pas des rapports ordinaires : issus d'un long travail de la société sur elle-même, ils en représentent le point d'orgue dont la Conférence de Rio rend compte. Le suivi de la feuille de route de l'Agenda 21 inaugure ensuite l'émergence d'un « espace public mondial » (Robelin, 2004:101-105) dont la dynamique jusqu'à aujourd'hui ne s'est pas démentie. 


\section{Moment 3 de la pelS : 1992-2014, la longue marche d'un mot méthode vers la globalisation d'un langage commun}

Rio de Janeiro met en route une dynamique qui fait du développement durable, du développement humain, du capital humain, de l'autonomie individuelle et de la logique écologique de la croissance quelques-unes des préoccupations majeures de tous les gouvernements du monde. Depuis, les grands rendez-vous se multiplient à toutes les échelles géopolitiques de la planète pour interpréter, traduire, mettre en forme et expérimenter les principes inscrits dans l'Agenda 21. Comme pour la conférence de Rio, chacun de ces rendez-vous s'appuie sur une série de textes intermédiaires prescripteurs et quelques grands textes fondateurs. Nous avons donc «surfé » dans l'immense production de ces documents issus des instances supranationales pour en isoler quelques-uns (encadré 2) : ceux qui jalonnent et accompagnent le voyage du mot méthode « investissement social » du début de l'aventure (1992 avec la Déclaration de Rio) à l'étape actuelle (le Rapport mondial du Pnud, 2014a).

Cette liste rend compte du travail nécessaire pour parvenir à un langage commun. Soutenue inlassablement par le réseau Pnud ${ }^{27}$, l'idée d'investissement social chemine, au point d'infiltrer progressivement tous les discours politiques accompagnant la mise en place des réformes économiques et sociales des pays et « régions » du monde entier (Deroussen, Collombet 2012). À la lecture de cette présentation rapide, on voit clairement que les derniers documents correspondent à une formulation de plus en plus intégrée et organisée de la perspective d'investissement social, à l'inverse des premiers, qui présentent une suite d'énoncés/définitions disparates qui cherchent à peine à entrer en résonance. Quand on sait que ces textes sont pour la plupart le résultat de processus de réflexion descendants puis ascendants, qui s'alimentent à toutes les instances traversées dont ce genre de processus est l'objet (partenaires institutionnels, acteurs de la société civile et du secteur privé, centres de recherche et universitaires à toutes les échelles de décisions (supranationales, régionales, territoriales, sans oublier les mobilisations luttes des mouvements démocratiques, les pressions de lobbies en tout genre, les rapports de force de la part de la Banque mondiale ou du Fonds monétaire international - FMI - et autres forces influentes comme peuvent l'être les grandes catastrophes climatiques, naturelles ou civiles), on peut faire l'hypothèse que ce qui est publié dans ces documents fait sens, la légitimation se construisant au rythme et en fonction des difficultés et des obstacles franchis. On peut donc dire qu'aujourd'hui la perspective d'investissement social s'institutionnalise, doucement mais sûrement.

27. Qui stimule le débat et en rend compte dans les rapports mondiaux annuels sur le développement humain $(\mathrm{RDH})$. 


\section{L'investissement social : de rapports en recommandations, la progression d'un mot méthode}

\section{2 : déclaration de Rio sur l'environnement et le développement}

La conférence de Rio de Janeiro officialise la formule « développement durable » et contribue au passage du développement strictement " économique " au développement dit " humain ». La déclaration de Rio alerte aussi les États de la nécessité de prendre en compte l'urgence des problèmes que posent les transformations du climat. La conférence propose ensuite une méthode non contraignante avec la mise en œuvre de l'Agenda pour le $\mathrm{XXI}{ }^{\mathrm{e}}$ siècle : des objectifs sont déterminés au niveau global, puis répercutés à l'échelle des États; d'autres procédures sont laissées aux initiatives locales ou nationales et se combinent à la procédure descendante. La conférence fixe enfin des objectifs à atteindre à horizon de dix, 15 ou 20 ans. Depuis cette conférence, plus de 140 pays ont publié quelque 600 rapports nationaux sur le développement humain avec le soutien du Pnud (Pnud, 2014a).

1993 : rapport Pnud sur le développement humain : la reconnaissance de la société civile à l'échelle mondiale, "la participation populaire »

Le rapport de 1993 du Pnud s'intéresse à trois grands moyens de participation populaire : des marchés orientés vers la personne, une gouvernance décentralisée et des organisations communautaires (en particulier des organisations non gouvernementales). II suggère aussi des mesures politiques concrètes pour résoudre les problèmes liés au nombre croissant de sansemploi et fait appel à la mise en place de règles définissant des modalités de participation). Une approche participative, (notamment à travers la participation des ONG) est dite indispensable à toute stratégie du développement humain réussi. Ce rapport peut être considéré comme une mise à la disposition de l'expérimentation des premières traductions de la dimension participative du développement durable, et ce en termes de stratégies montantes (topdown) ou descendantes (bottom-up) (Claval, 2006).

1998 ; 2001 : rapport OCDE (1998a) sur l'investissement dans le capital humain et rapport OCDE (2001) sur le rôle du capital humain et social

Suite à de longs errements, puis faiblement dès 1984, puis fermement à la fin des années 1990, l'OCDE s'exprime en faveur de la mise en place d'un nouveau " modèle de développement " ${ }^{1}$. La réflexion sur l'investissement dans le capital humain y occupe une place centrale et cet organisme a beaucoup contribué à organiser et rendre compte des débats des sociétés civiles pour " traduire » et enrichir le concept de " capital humain ». Aujourd'hui, bien au-delà de la simple éducation, ce concept ne cesse de recouvrir des dimensions multiples au point de permettre de lui préférer souvent celui de " capacité ", au sens d'Amartya Sen (à savoir, une disposition opportuniste à construire individuellement, empiriquement, institutionnellement dont tout individu doit être en possession à tout moment de son parcours de vie, au titre de « bien premier »; OCDE, 1998a, 2001). 
2000 : lancement des Objectifs du millénaire pour le développement

Au nombre de huit, les Objectifs du millénaire pour le développement (OMD) forment un plan proposé à tous les pays du monde et à toutes les grandes institutions mondiales de développement. En 2000, 193 pays les accepteront. Ces objectifs recouvrent de grands enjeux humanitaires : la réduction de l'extrême pauvreté et de la mortalité infantile, la lutte contre les épidémies, l'accès à l'éducation, l'égalité des sexes ou l'application du développement durable. On peut penser ce texte institutionnel comme le coup d'envoi du programme d'internationalisation des réformes dans la mesure où, depuis, « l'évolution la plus manifeste dans les politiques de développement est que les agendas lient désormais tous les pays, et non plus seulement les plus pauvres. Tous nos futurs sont réunis dans un écosystème global, climatique, économique, géopolitique ${ }^{2}$ ».

\section{9 : rapport de la Commission Stiglitz-Sen-Fitoussi sur l'évolution des indicateurs économiques}

La Commission Stiglitz-Sen-Fitoussi a remis en septembre 2009 un rapport sur la mesure de la performance économique et du progrès social et sur ses indicateurs. Un document qui s'appuie sur les très nombreux travaux de recherche dans les divers domaines des sciences économiques et sociales au cours des années 2000. Le rapport propose des indicateurs synthétiques de bien-être plus appropriés que le PIB, ainsi que des tableaux de bord visant à appréhender performance économique et qualité de la vie à travers leurs différentes facettes (Stiglitz, Sen, Fitoussi, 2009).

\section{2 : recommandation de l'OIT sur l'universalisation de la protection sociale}

Une initiative officielle des agences onusiennes recommande en juin 2012 l'« universalisation de la protection sociale » que le Pnud élargira en 1994 en parlant de prestation universelle de services sociaux de base. Elle fera l'objet la même année d'une recommandation de l'Organisation internationale du travail (OIT) qui envisage la sécurité sociale comme " un investissement dans les hommes et les femmes en leur donnant la capacité de s'adapter aux changements de l'économie et du marché du travail » (ILO, 2012). Passée relativement inaperçue et encore faiblement contraignante (sur le sujet, voir notamment Hirsch, 2011), cette initiative peut être comprise comme l'acception d'une méthode propre au développement humain durable, celle de l'investissement social. Cette perspective passe dans les pays en développement par la construction d'un « socle de protection sociale » et dans les pays développés par celle d'un État d'investissement social (AISS, 2013 ; Hirsch, 2011).

2013 : communication sur "les investissements sociaux en faveur de la croissance et de la cohésion " de la Commission européenne

Avec ce texte, la Commission européenne a adopté le 20 février 2013 une communication (Commission européenne, 2013) qui invite les États membres à donner la priorité aux « investissements sociaux » et à moderniser (ou créer) 
leurs systèmes de protection sociale. L'Union européenne propose ainsi un cadre d'interventions et, surtout, de révisions pour les politiques sociales des 27 États membres » (Boisson-Cohen, Pallier, 2014).

2014 : rapport sur le développement humain du Pnud (2014a). Pérenniser le progrès humain : réduire les vulnérabilités et renforcer la résilience

Le rapport sur le développement humain souligne l'importance de promouvoir les choix des personnes et de protéger les réalisations en développement humain. II soutient que la vulnérabilité est une menace pour le développement humain et que, à moins d'être " adressé de façon systématique ", le progrès ne sera ni équitable ni durable. L'objectif fondamental du développement n'est donc plus ici d'accumuler les biens et la richesse financière (société salariale de première modernité), mais d'investir socialement pour créer un environnement permettant aux individus de bénéficier d'une vie longue, en bonne santé et créative. En présentant la panoplie d'instruments de gouvernance expérimentés, ce rapport peut être considéré comme un temps fort des traductions disponibles de la dimension "écologiste " du développement, centré sur la figure d'un humain considéré comme une personne autonome, qui choisit son parcours, dans un environnement accompagnant et habilitant qui lui permet de dépasser et/ou d'éviter les obstacles tout au long de la vie.

\section{4 : rapport OCDE sur la gouvernance publique. Ensemble pour amé- liorer les services publics : partenariat avec les citoyens et la société civile}

Basé sur les résultats d'une étude préliminaire des pratiques nationales dans 22 pays membres de l'OCDE (au Brésil, en Égypte, en Russie ou en Ukraine), le rapport porte sur des approches novatrices et partenariales que les pouvoirs publics forment avec les citoyens, les usagers et les organisations de la société civile. Ces approches - dénommées co-production - font glisser la sémantique « services publics » vers celle de la " co-production des services au public » et promettent d'apporter des réponses créatives pour une meilleure qualité des prestations en période de restrictions budgétaires. " Big Society ${ }^{3}$ » sera la première grande opérationnalisation in situ de ces approches novatrices. Cette expérimentation anglaise sera peu convaincante et très critiquée ${ }^{4}$ mais, depuis, la perspective d'une production locale et collaborative de services au public suscite un intérêt croissant parmi les spécialistes et les praticiens.

1. La série des documents OCDE Perspectives de l'Emploi en attestent et notamment OCDE (1989, 1995, 1996, 1998b). Un rapport publié en 1999 (OCDE, 1999) est l'aboutissement d'une série de quatre forums internationaux sur le thème "L'homme, la nature et la technologie : des sociétés durables au XXIe siècle ".

2. Helen Clark, administratrice du Pnud, l'exprimait ainsi dans un article sur « Les grands enjeux du développement », Libération, 8 septembre 2015.

3. L'Angleterre a expérimenté cette piste dans quelques villes et quartiers en 2010 et abandonné le chantier en route sous les critiques issues de l'opposition travailliste et de la société civile. Depuis, d'autres modèles de services publics collaboratifs sont expérimentés dans le monde (Japon, Québec, Argentine, Espagne) et en France : voir Conseil économique, social et environnemental (2006).

4. " La Big Society ne fait que créer un profond cynisme : l'opinion y voit un subterfuge pour justifier les coupes budgétaires. [...] Le scepticisme sévit même au cœur du gouvernement, où certains fonctionnaires ont surnommé la Big Society "BS"... pour bullshit », Jacobs, Pickard, Parker (2011). 


\section{La perspective d'investissement social : la base d'une utopie réalisable?}

\section{II.1. Le socle commun de l'investissement social : la base d'une utopie réalisable?}

Dire que la perspective d'investissement social s'institutionnalise suppose une convergence sur un socle commun d'opérationnalisation. Est-ce aujourd'hui audible au regard de l'état du monde ? Non, mais en revanche, dire que la peIS avance est, de notre point de vue, audible dès lors qu'on reste sur ce registre très particulier du principe d'action dont les grands textes prescripteurs rendent compte.

À ce niveau de réalité, nous avançons donc l'hypothèse que la peIS propose un socle commun qui fait consensus et qu'il englobe trois piliers : la conception de l'objectif IS (la production d'un individu autonome), celle des moyens de l'IS (la redéfinition profonde de l'intervention publique) et celle du mode de financement de l'IS (l'activation). Nous allons résumer à grands traits ce que nous considérons comme l'essentiel des idées partagées.

\section{L'objectif : la production d'un individu autonome pour le développement et la croissance}

Produire un individu pour le développement et la croissance et non l'inverse

Si le rapport 1990 du Pnud invoquait comme objectif « le développement des choix et des libertés des personnes, par les personnes, pour les personnes », le rapport 2014 du Pnud le formalise nettement mieux en affirmant que l'objectif fondamental n'est plus l'accumulation des biens ni la richesse financière mais l'investissement dans « de meilleures politiques sociales et économiques ", " pour faire avancer les capabilités générales, améliorer directement la résilience humaine et permettre aux personnes de gagner en autonomie ». Dans ce cheminement, une conception nouvelle du développement s'affirme : il ne s'agit plus de faire de la croissance pour le développement du bien-être et des hommes mais l'inverse : il s'agit de développer le bien-être des hommes pour faire de la croissance. On assiste donc bien à une remise en cause (celle de la vision industrialiste et économiciste du développement) au profit d'une conviction commune (que le développement strictement économique n'arrive pas à bout du développement sociétal) et d'une prescription de plus en plus claire (celle de la perspective d'investissement social).

Cette grammaire humaniste du développement, on la retrouve aujourd'hui partout, des prescriptions en faveur de la responsabilité sociale des entreprises (RSE ${ }^{28}$ ) à celles en faveur d'un nouveau modèle d'État social (Esping-Andersen, Gallie, Hemerrijck et al., 2002), en passant par

28. La RSE est un Pacte mondial des Nations unies, relayé par des principes directeurs de l'OCDE, puis par la Commission européenne, puis par le rapport Brovelli, Drago, Molinier (2013). 
celles en faveur d'une société inclusive ${ }^{29}$. Dans toutes les feuilles de route, on ne prescrit plus aujourd'hui de produire (des biens manufacturés pour que l'homme puisse vivre, pour qu'il soit aidé et protégé) mais d'investir (socialement et économiquement dans la production de l'homme pour mettre à sa disposition une économie d'usages de biens et de services).

\section{Produire un individu dit « autonome »...}

En affichant comme objectif premier « la promotion des potentiels et des possibilités humaines et sociales » (Boisson-Cohen, Palier, 2014), la logique d'investissement social convoque une autre figure de l'individu en regard de celle inscrite dans le projet des régimes d'État-providence : un individu moins " assisté », plus " autonome ». On sait combien les conceptions contemporaines de l'autonomie sont nombreuses (Dworkin, 1988:6). Cela dit, dans ses principes d'action, la perspective IS s'accorde autour d'une conception nouvelle de l'autonomie. Dans cette perspective, on insiste d'une part sur l'idée que « toute approche visant à assurer et à pérenniser le développement humain doit être fondée sur la résilience, le but essentiel de la résilience étant de veiller à ce que l'État, la communauté et les institutions mondiales œuvrent à "autonomiser" les personnes » (Pnud, 2014a) et, d'autre part, sur l'idée qu'il faut en même temps «assurer le maintien d'un niveau élevé de protection sociale et d'augmenter l'autonomie des individu » (Delors, Dollé, 2009). La sémantique conjugue donc à la fois invocation à l'autonomie et injonction à la protection.

\section{... avec une vision participative de l'autonomie...}

Cette double dimension qui consiste à encourager à la fois la responsabilité de la personne et celle de la société renvoie, en toile de fond, aux nouvelles acceptions de la justice sociale. Dans cette perspective, on parle moins de "protection » (de l'individu pris en charge par la société) que de « contractualisation» (entre la personne et la société). Si les individus doivent être engagés, responsables, raisonnables (" être les agents positifs du changement au sein de leur communauté », "être en mesure de prendre part ${ }^{30}$ ", « mener la vie que l'on a des "raisons" de valoriser ${ }^{31}$ », etc.), en miroir, il est clair qu'ils doivent aussi être accompagnés (par la « mise en place de stratégies d'actions précoces visant à développer la résistance face à des situations difficiles et à renforcer la résilience » Pnud, 2014a, b). C'est donc une conception qui privilégie la figure d'un individu « actif » (c'est le « citoyen

29. L'inclusion sociale étant définie et mesurée à partir d'indicateurs sur le degré d'investissements et le développement des capabilités humaines : voir Conseil économique, social et environnemental (2014) ; Boisson-Cohen, Palier (2014).

30. « Être en mesure de prendre part à la société civile, aux services publics et aux processus politiques, et ce à tous les niveaux » (Delors, Dollé, 2009).

31. Car la personne est moins conçue " comme un individu rationnel que comme une personne raisonnable » (capable de coopérer avec les autres) et responsable (assumant des droits et des obligations vis-à-vis de son entourage), Mahieu (2006). 
qui développe ») mais qui ne reste pas seul face à l'adversité (ce sont des stratégies qui permettent). Autrement dit, c'est une conception « participative » de l'autonomie où la personne doit co-produire, avec l'Institution, les outils de résilience qu'exige toute sortie de crise dans le parcours individuel.

\section{... et une vision androgyne de l'autonomie}

Cette conception «androgyne » a été initiée par des représentantes féministes québécoises et reprise par le mouvement mondial des femmes ${ }^{32}$. Cette conception rejette les acceptions classiques de l'autonomie qui opposent l'accès à l'autonomie par le revenu (acception qualifiée de masculine) et la faculté de se déterminer par soi-même, de choisir et d'agir librement, de manière indépendante (acception qualifiée de féminine). Il s'agit ici de mettre en avant que les hommes et les femmes s'écartent à présent des stéréotypes de genre pour rejoindre une conception plus « androgyne ». Cette conception revendique « la possibilité d'avoir accès, au cours de l'ensemble des cycles de sa vie, aux moyens et ressources économiques (emploi, services, revenu suffisant) à la fois pour répondre à ses besoins (et à ceux dont on a la charge), pour avoir la possibilité de faire des choix économiques, et pour influencer les structures économiques de nos sociétés ${ }^{33}$ » et ce, tant pour les hommes que pour les femmes.

Ces conceptions participatives et androgynes de l'autonomie sont celles qu'on trouve dans les rapports et discours qui se réclament de la perspective IS, comme celles que l'on retrouve aujourd'hui dans tous les programmes d'intervention sociale préconisés par les grandes instances mondiales et régionales.

\section{Les moyens : la redéfinition profonde de la logique de gestion des risques et de l'intervention publique}

Comment se donner les moyens de produire cet individu autonome, fer de lance du développement et de la croissance à venir des sociétés humaines? Si l'on prend au sérieux la volonté de promouvoir les potentiels/possibilités humaines et sociales, on comprend que toutes les interventions, toutes les activités liées à la valorisation du capital humain (enseignement et formation, culture, santé, accompagnement social, services publics, etc.) doivent être interpellés et impliquent (de la part des institutions qui structurent et régulent le vivre ensemble) des manières de faire qui, pour la plupart des sociétés, restent à inventer. Ces orientations qui, on le comprend, engagent une forte dynamique de transformation du rôle des États dans et sur l'économie, se retrouvent aujourd'hui dans trois nouvelles logiques de gouvernance.

32. Fédération des femmes du Québec.

33. Argumentaire pour la campagne pour l'autonomie économique des femmes 2007-2008; Coordination du Québec de la Marche mondiale des femmes. http://www.ffq.qc.ca/2011/04/lautonomieeconomique-des-femmes-trousse-de-formation/. 


\section{Une logique de gestion et d'intervention centrée sur la personne}

Le grand basculement que va introduire l'approche-pro-active-centréesur-la-personne-autonome renvoie au choix des références de l'action publique par lesquelles les politiques seront conçues, mises en œuvre et évaluées. On retrouve le basculement dont on parle ici dans le choix de la formule omniprésente depuis une vingtaine d'années dans tous les agendas politiques mondiaux : «mettre l'individu au cœur de ». Toutes les réformes contemporaines se réclament en effet de vouloir remettre l'individu à sa place en le mettant : au cœur des dispositifs, au cœur de l'économie, des organisations, du système, des décisions, de l'entreprise, etc. Cette logique de gestion et d'intervention centrée sur la personne s'appuie sur deux piliers : l'individualisation du social et les nouvelles magistratures du sujet (Rosanvallon, 1983) :

- l'individualisation du social : c'est la proposition de reconstruire la notion de droit social autour d'une nouvelle conception du « sujet de droit». Dans cette perspective, le sujet de la protection et de l'intervention sociale ne sera plus l'individu mais la personne; et plus précisément encore la personne saisie dans sa trajectoire de vie. On peut considérer cela comme une réponse aux critiques des logiques d'intervention sociale dans les régimes d'État-providence ou dans l'aide au développement, qui accusent ces logiques de « ne pas savoir aider les individus » (Bresson, 2008). En se portant sur les situations complexes des personnes, la nouvelle approche du sujet de droit tente de quitter les catégories abstraites et universalistes ( l'homme moyen de Quételet $\left.{ }^{34} »\right)$ pour s'intéresser aux besoins singuliers des personnes concrètes.

- les nouvelles magistratures du sujet: la formule qui résume le basculement dont on parle ici est contenue dans une double injonction : pour le sujet ayant droit, il s'agit de l'injonction pour la personne à s'inscrire dans des cadres et des procédures « contractualisés »; et, pour les professionnels de l'action et de l'intervention sociale, il s'agit de l'injonction à « travailler autrement », non plus sur autrui mais avec autrui (ce qui signifie notamment de quitter la logique de standardisation des prestations qui s'attache à «l'état statique d'un individu » au profit d'une logique d'individuation qui s'attache à «la situation dynamique d'une personne »; Astier, 2009).

\section{Une logique de gestion et d'intervention proactive}

Proactive signifie à la fois équiper l'individu avant et l'équiper tout au long de la vie:

- équiper l'individu avant : la rupture de perspective entre la logique providentielle ${ }^{35}$ et celle de l'investissement social est dans le glissement d'une

34. « L'homme moyen de Quételet » renvoie à la moyenne statistique des comportements décrits par Adolphe Quételet. Cité par Rosanvallon (1995).

35. Nous appellerons « logique providentielle » la perspective contenue dans le référentiel d'action des États-providence d'après-guerre, qu'ils soient de type libéral (comme les États-Unis où l'État est faible) ou solidaire (comme la France, l'Allemagne ou les pays du Nord où l'État est fort). 
gestion des risques après-coup vers une gestion des risques « anticipative, systématique et intégrée du risque ». Dans la logique d'investissement social, l'idée ne sera donc plus de compenser le risque après coup (logique de correction/réparation) ni de le prévoir (au sens d'une prévention qui veut annuler l'idée même de danger en alertant sur ses conséquences négatives). L'idée d'IS, c'est de proposer de vivre avec le risque et d'équiper les gens en amont pour en limiter les conséquences (logique de transformation et de co-production des stratégies de résilience). Cette approche anticipatrice s'exprime dans les programmes de développement par un universel appel à concentrer les dépenses sociales sur l'enfance (le ciblage sur les femmes étant à comprendre d'abord comme un rempart contre la pauvreté des enfants dans un monde où les femmes isolées avec enfants est en constante progression).

- équiper l'individu tout au long de la vie ${ }^{36}$ : On l'a dit, travailler-avecla-personne signifie que la référence du sujet de droit n'est plus un individu abstrait mais une personne concrète dont la situation est pensée en rapport avec sa trajectoire de vie. Ne plus se référer à l'état de l'individu mais à sa situation revient à passer d'une approche catégorielle (passive ou statique) à une approche relationnelle (active ou dynamique) qui tient compte du contenu et du sens des parcours de vie (Nicole-Drancourt, 2007). Cette approche s'exprime par l'omniprésence de la clause « tout au long de la vie » qui accompagne la plupart des programmes d'aide au développement et des projets de réformes sur le droit à l'orientation, la formation, l'apprentissage, l'accompagnement, l'autonomie... Cette approche est à l'origine de la dimension proactive de la peIS : «protéger les individus TLV » ne signifie pas «toujours prévenir » mais « maintenir les trajectoires individuelles dans une succession de cycles de vie à vulnérabilité faible » en évitant aux personnes soit « de rester piégées dans un cycle à vulnérabilité forte » soit « d'entrer dans une mobilité à dynamique négative »; lutter contre la pauvreté ne signifie pas offrir un revenu de remplacement mais «lutter contre la vulnérabilité pour briser l'engrenage de la pauvreté ${ }^{37} »$. Dans ces glissements sémantiques où on ne parle plus « d'état de l'individu » ni de «pauvreté » de «personne dans un cycle de vie » et de «vulnérabilité » se cache un réel basculement des fondements d'une logique de protection sociale mais pas de sa disparition.

\section{Une logique de gestion et d'intervention participative et territorialisée}

Une logique de gestion proactive centrée sur la personne oblige à rapprocher individu et institutions, notamment géographiquement. Suite au rapport prescripteur du Pnud en 1993 sur « La participation populaire », de

36. TLV en français, life course perspective en anglais.

37. Ces basculements sémantiques sont particulièrement bien présentés dans un rapport officiel du BIT ; voir Bonilla Garcia, Gruat (2003). 
multiples expérimentations émergent et cherchent le cadre à même de faire participer les citoyens, les usagers et les organisations de la société civile à la fourniture de services publics (voire de services au public). S'appuyant sur ces expérimentations, l'OCDE conclut « qu'il importe que les pays facilitent le partage d'expériences à tous les niveaux de l'administration notamment à l'aide du partenariat entre administration centrale et collectivités locales » (OCDE, 2014:117). Cette orientation va accompagner la réforme des modes de gouvernance de deux manières :

- une tendance à la territorialisation des politiques publiques : ce processus doit valoriser des liens de proximité citoyens/institutions dans les endroits où ces liens sont défaillants (voire les revaloriser dans les sociétés où ces liens sont très abimés; Donzelot, 1996). À l'échelle nationale, on assiste donc, dans beaucoup de pays, à la recomposition des cartes territoriales et à la réaffectation des compétences : on tend à accorder une plus grande autonomie aux collectivités locales dans la gestion et dans la mise en œuvre des politiques sociales et, parallèlement, les États promettent de céder certaines de leurs prérogatives sociales à un tissu intermédiaire (associations, collectivités locales...) pour revivifier les solidarités de proximité (Lafore, 2004) ;

- un glissement de l'action publique vers la co-construction (ou co-production) : À travers l'injonction au partenariat (ou à la délégation contrôlée), l'idée qui fait son chemin est celle de repenser les modes traditionnels de prestations des services publics et sociaux en associant des nouveaux acteurs (habitants, usagers, représentants de mouvements, experts ou entreprises) et en renforçant la coopération avec des modèles de production minoritaires (coopératif, associatif, mutualiste, collaboratif, etc. ; Callon, Lascoumes, Barthe, 2001). Cette piste a été explorée dans divers pays sous des formes variées, le projet le plus abouti étant celui de «Big Society » au Royaume-Uni (voir supra, encadré 2).

Aujourd'hui, on retrouve ces nouvelles logiques de gouvernance partout, dans la constellation des réformes qui secouent à la fois l'ensemble des pays où l'État social est en crise et dans l'ensemble des pays où il est en construction... et on les retrouve aussi, exposées en détails, dans tous les documents qui présentent (ou analysent) la perspective d'investissement social (Delors, Dollé, 2009 ; Vielle, Pochet, Cassiers, 2005 ; Palier, 2014...).

\section{Le mode de financement : l'activation}

Toutes les sociétés doivent prévoir un financement de leur régime d'État social en adéquation avec leurs objectifs et le choix des moyens mis en œuvre pour les atteindre. L'État-providence l'a fait, la perspective d'IS le fait. Mais en passant d'une logique providentielle à une logique d'investissement social, le mode de financement du système va présenter à 
nouveau de profondes ruptures. L'expression sémantique de ces ruptures est contenue dans les notions paradigmatiques complexes comme « sécurité socioéconomique active » ou « sécurité proactive » ou tout simplement «activation ».

\section{Une nouvelle toile de fond au financement de la protection sociale : la société inclusive}

La toile de fond de tous les États-providence a été tissée autour d'un régime d'activité dit de plein emploi. Le plein emploi n'a pas grand-chose à voir avec sa définition intuitive puisqu'il a été conçu partout comme celui d'une main-d'œuvre masculine, plutôt adulte (Nicole-Drancourt, 2001). En effet, trempés à la sauce bismarckienne ou beveridgienne (pour les pays à forte régulation étatique) ou dilués dans des flots de conventions (pour les pays à faible régulation étatique), les rouages du paradigme plein emploi sont différents mais ont abouti dans tous les pays au même résultat : un logiciel « d'exclusion sélective » (Eyraud, Marsden, Sylvestre, 1990) aux commandes d'un marché du travail fortement andro-genré avec, d'un côté des plein-droits pour une population salariée restreinte (plutôt mâle et adulte), de l'autre des quasi-droits pour le conjoint inactif du salarié (plutôt des mères) et en « résiduel » des droits à l'assistance pour les « isolé(e)s » et autres en « incapacité » de travailler. La toile de fond (initiale) de tous les États-providence a donc été tissée autour d'un régime d'activité à géométrie variable avec d'un côté les actifs qui bénéficient pleinement du système et y participent pleinement et, de l'autre, les inactifs qui bénéficient du système mais $n^{\prime} y$ participentpas ${ }^{38}$. La toile de fond des sociétés d'investissement social sera tissée autour d'un régime d'activité différent. Dans ce nouveau régime, tout être humain doit avoir le droit d'accéder à l'autonomie par son travail : les hommes et femmes valides, mais aussi les mères et tous ceux que la logique providentielle exempte de recherche d'emploi pour cause d'inemployabilité ou d'incapacité ${ }^{39}$. L'IS va en effet afficher l'objectif d'une participation active du plus grand nombre à la société : elle parlera donc de moins en moins de plein emploi (trop connoté dans le paradigme de l'État-providence) au profit d'une autre notion, celle de société inclusive ${ }^{40}$. Si cette disparition de

38. La crise et le creusement croissant des déficits de la Sécurité sociale a bien sûr brouillé les cartes des frontières initiales des assiettes de recouvrements et de prestations : par exemple, en 1991 , une mesure fiscale apparaît (la CSG) pour combler les «trous » récurrents des caisses de la Sécurité sociale traditionnellement pourvue par des financements contributifs... et des inactifs bénéficiaires des minima sociaux la paient... Peut-on dire alors que depuis 1991 « tout le monde participe à financer le système ? " Je répondrais que non car cette mesure reste une mesure non cohérente avec la logique providentielle. La CSG paie le système mais ne fait pas système...

39. Cette thématique de l'augmentation des taux d'emploi est à nouveau la thématique majeure au programme du Forum de l'OCDE qui tous les ans depuis 15 ans réunit chefs d'État, dirigeants d'entreprises, ONG, syndicats et experts : http://www.oecd.org/fr/forum/pour-un-marche-du-travail-inclusif.htm.

40. Issus du mouvement pour l'accessibilité universelle et l'intégration des personnes handicapées, l'idée de "société inclusive " se répand au rythme où les populations victimes d'exclusion sociale s'en emparent pour lutter " contre » (contre les discriminations, contre le racisme et autres dénis de reconnaissance). La notion de société inclusive s'imprime alors au menu des événements de divers espaces publics. Voir « Investir dans les solidarités pour construire une société inclusive », États généraux de l'Économie sociale et solidaire, 17-18-19 juin 2011. 
la « citoyenneté au rabais » est une promesse qui peut séduire, l'IS ne la revendique pas pour autant comme telle, l'enjeu étant ailleurs, à savoir, le financement de son modèle. La peIS ne s'en cache d'ailleurs pas : «Nous avons besoin de plus d'actifs de tout âge pour financer nos dépenses sociales à mesure que notre population vieillit et, donc la participation de tous au travail participera à la création de valeur par tous » (Wim, 2003). Cela dit, en abandonnant le principe « d'exclusion sélective » de la logique providentielle (aujourd'hui coûteuse, contreproductive et stigmatisante), l'IS va résoudre l'équation de son financement en misant sur des valeurs plus vertueuses et plus en phase avec les aspirations d'émancipation contemporaines (citoyenneté de plein droit, reconnaissance, utilité sociale).

\section{Un nouveau logiciel de transferts financiers : l'activation des dépenses passives ${ }^{41}$}

Mettre et/ou remettre «tout le monde au travail », augmenter les taux d'emploi, construire un marché du travail inclusif ne relève pas de la génération spontanée et nécessite la médiation d'un outil incitatif. Cet outil sera l'activation. Partant des acquis des États-providence (ou inventant des dispositifs dans les pays où un système de protection sociale se met en place), le principe d'activation aura partout pour dénominateur commun celui du « making work, pay » (Barbier, Sylla, 2006), autrement dit s'adossera à l'idée que toute heure travaillée doit rapporter au travailleur plus qu'une heure « indemnisée » par le système de protection sociale des « exemptés du travail ${ }^{42}$. Cette incitation financière est conçue à la fois pour augmenter les taux d'emploi mais aussi pour accélérer le processus de formalisation du travail (Bouffartigue, Busso, 2011) : une personne active en plus, une activité qui sort de l'informalité, un emploi qui sort du travail au noir c'est autant de possibilités pour le système de prélever une part de "valeur » pour alimenter le financement de l'ensemble.

Au-delà du raisonnement comptable, l'activation est un principe de financement qui bouscule fortement les fondements de l'architecture du système de protection sociale de type providentiel. En effet, la perspective IS ne conçoit plus d'un côté le travail (qui permet un revenu) et de l'autre la protection sociale (qui garantit un maintien du revenu hors activité). Avec l'objectif prioritaire de produire une personne autonome équipée pour travailler selon ses capacité tout au long de la vie, l'IS ne distingue plus travail et protection sociale puisqu'il conçoit l'emploi en termes de «solution universelle » dans le traitement social des problèmes (Barbier, 2001). Ce glissement s'affichera dans les travaux des législateurs européens par un slogan, « mettre l'emploi au cœur de la protection sociale». Mettre l'emploi au

41. Pour une vue d'ensemble et pour accéder aux travaux sur la mise en place, les expérimentations et l'évaluation des politiques d'activation des dépenses passives à travers le monde, voir l'étude thématique de l'OCDE : www.oecd.org/els/emploi/pamt.

42. En France, le premier affichage de l'adoption de cette stratégie s'est exprimé par le rapport dit Pisani-Ferry (2000). 
cœur de la protection sociale n'est pas nouveau dans les économies d'Europe continentale dont les régimes de protection sociale sont de nature bismarckienne (Esping-Andersen, 1990/1999). Ce qui l'est en revanche, c'est que, dans la perspective d'investissement social, la relation emploi et protection sociale est inversée : dans le modèle bismarckien, l'emploi fait accéder à la protection sociale alors que, dans l'État d'investissement social, la protection sociale est ouverte à tous et doit être organisée de façon à conduire ou à reconduire l'emploi (Delors, Dollé, 2009).

Le principe d'activation a fait, fait et fera encore longtemps l'objet de sévères critiques : cet « intéressement » à l'engagement professionnel (compris par de nombreux experts comme une contrepartie) n'est-il pas le cheval de Troie d'un processus de destruction en règle de tout l'État social ${ }^{43}$ ? Sans entrer dans un débat qui n'est pas l'objet de cet article, on peut néanmoins préciser cela : comprise dans la perspective " providentielle », l'activation est dangereuse et contraire aux acquis sociaux ; mais comprise dans la perspective d'investissement social, force est de constater que l'activation peut être présentée comme un instrument de préservation de ces acquis dans la mesure où elle finance la cohérence, la logique et l'objectif de développement des sociétés s'en réclamant.

\section{Des traductions de I'IS à géométrie variable}

Le socle commun du principe d'investissement semble se traduire différemment en fonction du degré de formalisation du travail ${ }^{44}$ et/ou des niveaux de développement du capital humain ${ }^{45}$ des pays concernés.

Dans les pays où la formalité du marché du travail est forte (Dufour, Jenson, 2003), où le niveau de vie est élevé et la main-d'œuvre fortement qualifiée, le principe d'investissement social va se concentrer sur l'enfance, la jeunesse et l'objectif de formation tout au long de la vie. Son financement s'exprime plutôt par une surenchère de la rhétorique d'insertion, surtout dans les pays où le chômage est endémique : il s'agira alors de lutter contre les trappes à inactivité, de multiplier les dispositifs de "retour à l'emploi » et de mise au travail des inactifs (ceux « éloignés de l'emploi » ou ceux « exemptés du travail » que la logique providentielle légitimait et dont elle a permis l'explosion à l'occasion des ratés du filet de sécurité). Cette rhétorique de l'insertion s'adossera néanmoins à des dispositifs de type nouveau, qui relèvent de l'investissement social : en France, des mesures

43. Sur la question, la littérature est abondante et les colloques traitant de la " fin de l'État social » se sont multipliés au cours de la décennie 2000. Voir notamment Bec (2007), et le colloque international à l'Université Paris 1 Panthéon-Sorbonne des 11, 12 et 13 septembre 2006 ayant pour thème "État et régulation sociale ".

44. Voir le classement des pays en fonction des degrés de formalité de leurs marchés des produits et du travail (Banque mondiale, 2013).

45. Voir l'indice de développement humain (IDH) du Pnud, mesure de synthèse du niveau moyen atteint dans les dimensions clés du développement humain : une vie longue et saine, l'acquisition de connaissances et un niveau de vie décent. 
phares comme la création des crédits d'impôts/prime pour l'emploi (PPE), comme le revenu de solidarité active (RSA), le contrat de transition professionnelle (CTP) ou la prestation partagée d'éducation de l'enfant (PreParE) en sont l'expression.

En revanche, dans les pays où la formalité du marché du travail est faible, où le niveau de vie est diversement distribué (voire faible) et où la main-d'œuvre est peu qualifiée, le principe d'investissement social va se concentrer sur l'enfance, les mères pauvres, en s'appuyant sur le vecteur de " capacité à exercer un pouvoir » (i.e. empowerment). Cela va s'exprimer par l'activation des minima sociaux mais, en plus, par la promotion des transferts monétaires conditionnels en direction des plus pauvres (Conditional cash transfers ou CCT) (Fiszbein, Schady, 2009 ; pour un regard critique sur les CCT, voir Revue Tiers Monde, 2013), les CCT étant la figure forte de l'IS dans la lutte contre la pauvreté via des politiques publiques qui encastrent mesures d'éducation, mesures de santé, politiques d'insertion et autonomie des mères. Ces politiques se compléteront par des investissements à objectifs sociaux du type création de petites et moyennes entreprises, accès au micro-crédit, construction ou réhabilitation d'infrastructures locales par des coopératives de travail (Trenta, 2014 ; des programmes qu'on verra d'ailleurs en augmentation notable dans nombre de pays dits riches, en direction des populations très appauvries par l'ampleur des restructurations industrielles, des récessions économiques et du chômage). Une des fortes spécificités de l'IS dans les pays où la formalité du marché du travail est faible, c'est qu'elle ne trouve pas les moyens financiers de ses objectifs : dépendant de leurs financeurs, les pays dépendants sont donc sous tension perpétuelle, ce qui ouvre la voie à de sévères luttes d'influence entre les organismes de développement (notamment le Pnud), les créanciers (Banque mondiale ou FMI) et les pays endettés (Michaud, 2007 ; Bénicourt, 2005). Les dérives et critiques que connaissent les pratiques de micro-crédit ou les programmes CCT (Ceballos, Lautier, 2013) en attestent.

\section{II.2. L'investissement social : un nouvel imaginaire social pour penser l'ordre du genre?}

On l'a déjà évoqué, la perspective IS divise. On l'a dit aussi, ces critiques ne sont pas fausses en soi, car à l'horizon de la perspective d'investissement social est à la fois la fin de la perspective providentielle de l'État social en général, la fin du pacte social collectif qui en est issu en particulier. Autrement dit, si les critiques se réfèrent au fait que les réformes en cours sont en contradiction avec l'équation sociale providentielle, elles ne sont pas fausses. Ce qui l'est en revanche, c'est de « lire » ces réformes de l'IS dans le paradigme providentiel alors qu'elles renvoient à un autre paradigme, celui 
de l'investissement social. Faire cela pousse à la confusion des registres de comparaison et à la confusion tout court.

Car, si l'on lit les réformes en cours dans la logique de conception de l'investissement social, que peut-on en dire? Nous proposons de développer rapidement un argument dans ce sens dans un domaine : celui de l'ordre social genré.

\section{La perspective d'investissement social : une « sensibilité certaine au genre »}

La déclaration de la quatrième Conférence mondiale sur les femmes organisée à Pékin en 1995 présente un programme pour l'autonomisation des femmes considéré comme le premier document de politique mondiale en matière d'égalité des sexes. Grâce aux travaux académiques des women studies ${ }^{46}$ et aux mouvements sociaux féministes ${ }^{47}$, toutes les instances de régulation mondiales promettent à présent une approche intégrée (nommée en Europe "gender mainstreaming»), qui «évalue les incidences pour les femmes et pour les hommes de toute action envisagée (notamment dans la législation, les politiques ou les programmes, dans tous les secteurs et à tous les niveaux) [...] et incorpore les préoccupations et les expériences des femmes aussi bien que celles des hommes dans l'élaboration, la mise en œuvre, la surveillance et l'évaluation des politiques et des programmes dans tous les domaines politique, économique et social ${ }^{48} »$. La perspective d'investissement social n'a pas manqué de prendre le train en route : frileuse à l'origine en encourageant la simple "participation des femmes aux stratégies de développement », la perspective IS s'est ensuite repositionnée beaucoup plus fermement ${ }^{49}$.

- À l'échelle globale, au nom de la prise de conscience du «pouvoir économique et social » des femmes, les politiques d'investissement social accordent une place centrale à l'approche intégrée pour penser les programmes d'éducation, la transformation des structures familiales ou l'évolution des régimes d'activité (Duflo, 2003). Mobilisant les outils de l'activation (programme de transferts monétaires, d'autonomie par le travail), ciblant les obstacles à l'insertion (par des programmes pour l'accueil des enfants et de services aux familles), toutes les prescriptions internationales encouragent la hausse des taux d'emploi féminin, les formes d'emploi et d'organisation du temps de travail favorables à la conciliation travail/

46. Notamment ceux issus des sciences humaines et sociales, des sciences politiques et des sciences juridiques.

47. Qui s'exprime à l'échelle supranationale à travers des institutions comme la Marche mondiale des femmes : http://www.mmf-france.fr/quinoussommes.htm.

48. Définition ECOSOC 1997.

49. Les travaux de Gösta Esping-Andersen sur les Régimes d'États-providence ont été à l'origine de nombreuses critiques de la part des women studies. Dans les années 1990, les travaux de Jane Lewis, d'Ann Orloff, de Diane Sainsbury ou de Rose Mary Crompton ont alors fait autorité. Parmi ces travaux, notons : Lewis (1998), Orloff (1993), Sainsbury (1994). 
famille, l'égalité de traitement, l'accès à la formation pour tous, la suppression des écarts de rémunération, etc.

- À l'échelle européenne, dès 2001, les instances européennes mettent en avant le « gender mainstreaming» et la lutte contre les inégalités de genre dans l'orientation des directives (Esping-Andersen, Palier, 2008 ; Palier, 2005). Dès 2003, le Conseil européen invite la Commission à lui rendre compte annuellement des progrès accomplis pour promouvoir l'égalité et des orientations en vue d'intégrer la dimension hommes/femmes dans tous les programmes politiques.

- Enfin en France, après une succession de lois pour améliorer l'égalité entre hommes et femmes au travail et en famille, le "gender mainstreaming » a été opérationnalisé dans tous les secteurs des politiques publiques. Cette démarche s'est soldée en 2014 par une loi (la loi cadre pour l'égalité réelle entre les hommes et les femmes ${ }^{50}$ ) présentée comme un pas inédit vers l'effectivité des droits.

On peut donc dire qu'aujourd'hui l'on retrouve dans les programmes de développement de tous les pays une « sensibilité au genre » certaine ${ }^{51}$.

\section{Une « sensibilité au genre " ou une " prise en compte des rapports sociaux de sexe »?}

Si la perspective d'investissement social a d'abord intrigué la mouvance féministe, il semble que l'engouement soit retombé pour laisser place à de sévères critiques voire à une franche hostilité. Une des premières voix à porter cette perspective (Dufour, Jenson, 2003), Jane Jenson, finit alors par douter puis par critiquer ouvertement ce qu'elle considère, avec d'autres, comme un détournement de cause : la perspective IS se serait progressivement « détournée de la question des femmes adultes pour se focaliser sur la situation des filles et sur la santé maternelle » (Jenson, 2011), et ce faisant combinerait des mesures égalitaires (pour les filles) à des mesures maternalistes (pour leurs mères) et occulterait ainsi l'égalité des sexes, «l'une des plus importantes revendications d'égalité mises en avant par les féministes depuis des décennies »(Jenson, 2011, op. cit.). Cette critique met donc en cause tant les programmes de transferts monétaires des "pays en développement » (Molyneux, 2007) (qui ciblent les mères) que ceux issus du recentrage du traité de Lisbonne (Jenson, 2011 op. cit.) (qui ciblent l'investissement en direction des enfants, l'augmentation des taux d'activité féminin et celle des services en direction des familles).

La perspective d'investissement social afficherait donc une certaine « sensibilité au genre » mais ne prendrait pas en compte les « rapports sociaux de sexe», ce qui plomberait toute perspective d'égalité des sexes. On

50. Loi préparée par le Rapport Lemière (2013).

51. Pour reprendre l'expression de Jane Jenson (2011). 
pourrait donner crédit à ce soupçon si, par ailleurs, ces courants critiques ne pensaient pas l'égalité des sexes comme un objectif politique à inscrire aux programmes. Or de notre point de vue, l'égalité des sexes est du registre de l'axiologique et à ce titre ne relève pas du contenu des agendas politiques qui peuvent proposer des programmes de politiques sociales et/ou économiques non conformes aux idéaux mais qui participent à tendre vers eux.

\section{Mesures féministes égalitaires ou nouvelles relations instituées non hiérarchiquement sexuées ?}

Cette question aux accents sibyllins a été posée dans le sillage des travaux comparatifs des États-providence par les féministes elles-mêmes. Alors qu'un consensus s'affichait pour classer ces régimes comme " protecteurs pour les femmes » (women-friendly), les travaux féministes ont cassé l'enthousiasme en montrant d'une part que les États-providence reposaient et reposent encore « sur un modèle sexué d'accès aux droits sociaux qui définit et traite les femmes en tant qu'épouses et/ou mères » (Lewis, 1995); et d'autre part que les femmes peuvent être l'objet des politiques publiques (et même bénéficier d'une certaine générosité publique) sans être pour autant intégrées à égalité avec les hommes dans la citoyenneté sociale et politique (Jenson, Lépinard, 2009). Autrement dit, ces travaux montrent que derrière des mesures politiques d'égalité des sexes persistent des "injustices ancrées dans la société et encodées dans les protections sociales » (Fraser, 2010).

Avec sa théorie de l'émancipation (Fraser, 2010, op. cit.), Nancy Fraser montrera bien l'erreur à penser l'égalité des sexes en termes de politiques d'action. Pour elle, éthiquement, il faut hisser la question de l'égalité des sexes au registre des droits humains mais, politiquement, il faut partir des « arrangements sociaux institués » pour s'affranchir des formes de sujétisation ancrée dans la société. Les droits sociaux sont alors à analyser comme des remèdes transformateurs ou simplement correcteurs et ce, quel qu'en soit le contenu. Les remèdes correcteurs visent à compenser les résultats inéquitables de l'organisation sociale sans toucher à leur cause profonde ; les remèdes transformateurs visent les causes profondes qui fondent l'ordre social en déconstruisant les mécanismes de production et de reproduction de cet ordre et, pour Fraser, seuls les remèdes transformateurs sont à même de produire l'innovation sociale. Autrement dit, si l'enjeu est de reconstruire une relation institutionnalisée (par des outils à géométrie variable) dans un sens moins injuste du point de vue du sens de la progression souhaitée (l'égalité des sexes), toute réforme doit être jugée dans le registre de son enjeu et non au regard de son objectif lointain. Nous pensons que dans ses principes et dans certaines réformes en cours qui s'en réclament, la perspective d'investissement social est « féministe » dans la mesure où beaucoup de programmes (dans leur mise en forme et/ou dans 
leur mise en œuvre) agissent, dans une dynamique transformatrice, pour la construction de relations égalitaires entre hommes et femmes. Autrement dit, là où certains critiquent l'absence de politiques d'égalité des sexes, nous avons envie de saluer l'ouverture de voies vers de nouvelles relations instituées, non hiérarchiquement sexuées.

\section{L'IS ou la promesse de nouvelles relations instituées non hiérarchiquement sexuées?}

Ayant récemment travaillé sur ces questions, je propose pour illustrer cette position trois exemples issus de la perspective d'investissement social en Europe.

\section{L'IS rompt avec la logique de l'aide aux mères travailleuses}

Au nom de l'« égalité entre hommes et femmes », les politiques des pays européens «women-friendly » ont misé sur les dispositifs d'aide aux travailleuses (avec le développement des crèches ou celui du temps partiel où elles sont prioritaires et majoritaires) et, ce faisant, ont non seulement conduit les femmes à se responsabiliser seules sur l'activité domestique et familiale mais ont aussi conduit les hommes à s'éloigner d'une prise de conscience et à renforcer leur carrière ${ }^{52}$. Dans le régime d'activité, qui envisage une participation de tous aux activités de la société et un accompagnement au retour à l'emploi des mères en rupture d'activité, la perspective d'investissement social entend relever les taux d'emploi féminin à hauteur des taux d'emploi masculin moins par l'injonction du droit à l'emploi des femmes (registre de l'axiologique) que par celle de développer des services d'accueil, de garde d'enfants, d'accompagnement à la dépendance tout en « activant» les prestations des politiques familiales en direction des pères, des mères et des parents isolés (registre du politique). En Europe, cette orientation n'est pas maternaliste mais féministe, comme l'est, dans les pays en développement, la décision d'inciter tout choix émancipateur (qui vont de se soigner, d'envoyer un enfant à l'école ou de se former pour s'insérer professionnellement).

Pourquoi féministe? Avec ses orientations d'IS, l'Europe prend d'abord conscience que la décision pour les femmes d'accepter un travail rémunéré ne dépend pas que de l'attractivité d'un emploi mais dépend aussi et surtout de leur disponibilité, le principal obstacle à l'égalité entre hommes et femmes au travail n'étant pas le droit à travailler mais l'assignation prioritaire des mères aux responsabilités familiales (qu'elles soient actives ou non actives) qui dévore la disponibilité de leur force de travail. L'Europe prend ensuite acte d'agir par des politiques transformatrices : la dimension transformatrice est contenue dans ce qu'elle appelle une " approche holistique des services » (Letablier, 2007) qui promeut la nécessité d'établir

52. Phénomène que Pascale Vielle (2002) appelle le « double renforcement ». 
une approche intégrale et pluridisciplinaire associant toutes les politiques sectorielles concernées vers une meilleure conciliation des temps travaillés (professionnels et domestiques) pour tous. On passe d'une logique «d'aide aux mères travailleuses » (logique correctrice) à une logique « d'aide à la conciliation pour tous » (une orientation qui porte en elle une dimension transformatrice de l'ordre genré).

\section{L'IS rompt avec la dualisation sexuée des parcours de vie}

Dans la perspective d'investissement social, l'enjeu est de maintenir toute personne dans une situation qui garantisse une capacité d'autonomie par le travail tout au long de la vie. Et ce, pour les hommes et pour les femmes. Cela ne signifie pas que tout le monde travaille tout le temps à plein temps tout au long de la vie mais plutôt que tout le monde reste en état professionnel tant qu'il en est capable, l'objectif principal de l'IS étant de le permettre par des mesures qui vont de la sécurisation des parcours de vie (flexicurité), à l'offre de congés parentaux de types nouveaux, en passant par la certification de compétences issues de la vie quotidienne. Cette orientation s'approche d'une utopie, celle de « réinventer un salariat qui défende l'utilité sociale du travail» et procure au travailleur une «nouvelle disposition de sa force de travail » (Fitoussi, Rosanvallon, 1998). Cette sémantique traduit une " politique des espaces-temps » (Viriot Durandal, 2004) de type nouveau qui impose implicitement de structurer l'organisation des parcours de vie dans d'autres perspectives (pluriactivité) avec d'un côté un rééquilibrage entre les différents domaines de l'existence (entre vie professionnelle, vie sociale, vie privée et familiale) et de l'autre la reconnaissance de ce rééquilibrage par des supports légaux (des droits et protections nouveaux à intégrer au rapport salarial). Si l'on s'accorde pour penser que la perspective d'investissement social va dans ce sens, alors une rupture essentielle est à l'œuvre de notre point de vue : par la reconnaissance d'un droit d'accès au travail pour tous, par celle d'un droit d'accès direct à la protection sociale pour tous et par celle d'un accès au droit qui passe par le travail et nécessite un "état professionnel permanent » tout au long de la vie, l'IS met en cause l'ordre sexué et hiérarchisé des régimes temporels fordistes (Nicole-Drancourt, 2013). Avec l'IS et ses référentiels d'action, un vaste phénomène d'hybridation, de complémentarité et de contamination des temps sociaux se met à l'œuvre : ces enchevêtrements associent d'un côté les divers temps sociaux (travail, activité familiale, formation, activités civiques) avec diverses logiques de sécurité économique (assurance, aide sociale, fiscalité, auto-production) qui bousculent la traditionnelle discipline de travail et partition des activités basée sur la division sexuelle. Avec l'IS, on passe donc d'une « masculinisation des trajectoires d'emploi des femmes" (logiques correctrices) à une "féminisation des parcours de vie de tous » (logique transformatrice vers un nouvel ordre genrê). 


\section{L'IS ou la mise en risque du care dans le rapport salarial}

En faisant de la trappe à inactivité le risque numéro un du basculement vers un cycle de vulnérabilité, la perspective d'investissement social permet de «mettre en risque » d'autres catégories de l'existence (jusqu'alors exclues de la relation salariale) et permet de les définir en termes de nouveaux droits sociaux. En effet, mettre l'emploi au cœur de la protection sociale permet de penser (pour les hommes comme pour les femmes) tout risque de déstabilisation (ou de non-réalisation) de carrière professionnelle en termes de perte d'autonomie, et ouvre un droit à préserver sa capacité de retour vers l'emploi. Cela permet donc de penser l'activité familiale (fort déstabilisateur de trajectoire professionnelle) en termes de risque économique pour l'individu, comme cela permet de penser l'activité professionnelle continue (fort vecteur de renoncement à la paternité ou à la maternité, fort obstacle au rôle d'aidant) en termes de risques économiques pour la société. Dans ce cadre, l'activité de care n'est plus pensée en termes de gêne à la disponibilité des travailleurs ni «compensée » par des mesures de protection, mais pensée en termes de nécessité au service de la reproduction de l'espèce dans laquelle toute personne (et non seulement les mères) détient un intérêt. Ce service pour tous est reconnu pour ce qu'il est (une nécessité) mais aussi pour ce qu'il menace (la capacité d'autonomie) et consacre alors une rupture à de multiples niveaux : d'une part elle rompt, par définition, avec l'affectation sexuée et andro-hiérarchisée des activités socialement utiles ; d'autre part, elle s'attaque à la "dualisation» de la citoyenneté (citoyenneté de " plein droit » versus citoyenneté « au rabais ») ; enfin, au moment où le vieillissement de la population et le choix du maintien à domicile s'imposent, elle permet d'envisager des pistes pour impliquer l'ensemble de la société (et non seulement les femmes) à accompagner ses dépendants en général (et ses aînés en particulier). Dans ces perspectives, il s'agit moins de soutenir une prise en charge par les femmes (mères, filles, servantes) de tâches qui leur seraient assignées que d'accompagner « les solidarités familiales » en renforçant l'encadrement légal par des nouveaux droits. Autant de propositions vers des droits à la reconnaissance des activités de care, vers la légitimité de les prendre en charge sans distinction de sexe et vers la protection des prestataires. Avec l'IS, on passe donc d'un renforcement des compensations des intermittences d'emploi liées à la prise en charge du care (logiques correctrices) à la «mise en risque » du care dans le rapport salarial (logique transformatrice vers un nouvel ordre genrè).

\section{Conclusion}

Le chemin est long des conceptions à leur réalisation : si le mouvement démocratique est en passe de gagner la planète, les conflits, les guerres, 
les grands risques environnementaux et climatiques nous rappellent que l'avenir reste incertain. Cela dit, rien ne permet de croire que la perspective d'investissement social a été une mode, un engouement passager et qu'elle restera sans lendemain. Dès lors, il est important d'y réfléchir, de la présenter, de la discuter, de la critiquer et, pourquoi pas aussi de chercher ce qui pourrait légitimer qu'on s'y réfère pour penser le changement vers un nouvel imaginaire social et démocratique. C'est du moins cette conviction qui fut à l'origine de cet article. Notre argumentaire a tenté de montrer qu'à partir de quelques pièces d'un puzzle complexe, on peut dégager un sens au chantier d'ensemble. Nous avons donc tenté de montrer : qu'en dépit d'un manque de visibilité, la perspective d'investissement social a fait son chemin, s'est frottée à l'épreuve des faits et s'est imposée au point de devenir une option sérieuse pour un imaginaire collectif alternatif ; que sous des expressions diverses liées aux chemins de dépendance nationaux et régionaux, la peIS présente aujourd'hui une base solide, cohérente et consensuelle dans ses principes d'action; enfin que toute réforme devant être resituée dans la cohérence du paradigme dont elle se réclame, les réformes en cours sont plus que jamais aujourd'hui à être resituées dans le paradigme d'investissement social. 


\section{Références bibliographiques}

AISS (2013), Sécurité sociale dynamique : un engagement global pour l'excellence. Développements et tendances, Rapport global 2013, novembre.

Astier I. (2009), « Les transformations de la relation d'aide dans l'intervention sociale », Informations sociales, $\mathrm{n}^{\circ} 152$, p. 52-58.

Banque mondiale (2013), Risques et opportunités : la gestion du risque à l'appui du développement, Rapport sur le développement dans le monde 2014.

Barbier J.-C. (2001), « Europe sociale : l'emploi d'abord », Quatre pages, n 44 , CEE, mars.

Barbier J.-C., Sylla N.-S. (2006), « Une synthèse de la littérature à propos de l'activation », in Barbier J.-C. (2006), Analyse comparative de l'activation de la protection sociale en France, Grande-Bretagne, Allemagne et Danemark, CEE, Rapport de recherche pour la Dares dans le cadre des lignes directrices de la Stratégie européenne pour l'emploi, janvier, p. 13-34.

Bec C. (2007), De l'État social à l'État des droits de l'homme ?, Rennes, PUR.

Beck U. (1986/trad. fr. 2001), La Société du risque : sur la voie d'une autre modernité, Paris, Aubier.

Becker G.S. (1964), Human Capital, A Theoretical and Empirical Analysis with Special Reference to Education, New York, Columbia University Press for the National Bureau of Economic Research.

Bénicourt E. (2005), Les analyses du PNUD et de la Banque mondiale sur la pauvreté et le développement: la place d'Amartya Sen, Thèse de doctorat en socioéconomie du développement, EHESS.

Boisson-Cohen M., Palier B. (2014), « Un contrat social pour l'Europe : priorités et pistes d'action » Note d'analyse, n¹9, France Stratégie, décembre.

Bonilla Garcia A., Gruat J.-V. (2003), Protection sociale : investir à toutes les étapes du cycle de vie pour la justice sociale, la réduction de la pauvreté, et le développement durable, Secteur Protection sociale, BIT, Genève, novembre.

Bouffartigue P., Busso M. (2011), « Précarité, informalité : une perspective NordSud pour penser les dynamiques des mondes du travail », Les mondes du travail, n 9-10, p. 27-39.

Boyer R. (2015), Économie politique des capitalismes. Théorie de la régulation et des crises, Paris, La Découverte.

Bresson M. (2008), "L'individu dans les modes de catégorisation du social », Informations sociales, $\mathrm{n}^{\circ} 145, \mathrm{p} .136-147$.

Brovelli L., Drago X., Molinier E. (2013), Responsabilité et performance des organisations. 20 propositions pour renforcer la démarche de responsabilité sociale des entreprises (RSE), Rapport public au Gouvernement, juin.

Callon M., Lascoumes P., Barthe Y. (2001), Agir dans un monde incertain. Essai sur la démocratie technique, Paris, Éditions du Seuil.

Castel R. (1995), Les métamorphoses de la question sociale. Une chronique du salariat, Paris, Fayard.

Ceballos M., Lautier B. (2013), « Les CCTP en Amérique latine, entre modélisation internationale et conjonctures politiques nationales », Revue Tiers monde, $n^{\circ} 214$, p. 219-245.

Chapel S. (2008), "L'imaginaire selon Cornélius Castoriadis », La Vie des idées, 26 novembre. 
Claval P. (2006), « Le développement durable : stratégies descendantes et stratégies ascendantes ", Géographie Économie Société, vol. 8, n 4, p. 415-445.

Commission européenne (2013), Communication de la Commission au Parlement européen, au Conseil, au Comité économique et social européen et au Comité des régions, Investir dans le domaine social en faveur de la croissance et de la cohésion, notamment par l'intermédiaire du Fonds social européen, au cours de la période 2004-2010, COM/2013/83 final, Bruxelles, 20 février.

Conseil économique, social et environnemental (2006), Aménagement du territoire, services publics et services au public, Rapport présenté par Jean-Alain Mariotti au CESE, 18 janvier.

Deacon B. (2001), « International Organisations, the EU and Global Social Policy», in Sykes R., Palier B., Prior P. (eds.), 2001, Globalization and European Welfare States: Challenges and Changes, Londres, Macmillan. Trad. fr. : "Les organisations internationales, I'Union européenne et la politique sociale globalisée », Lien social et politique-RIAC, $\mathrm{n}^{\circ} 45$, p. 73-87.

Debonneuil M. (2010), L'économie quaternaire : une croissance durable à construire, Rapport pour le Centre d'analyse stratégique, janvier.

Delors J., Dollé M. (2009), Investir dans le social, Paris, Odile Jacob.

Deroussen J.-L., Collombet L. (2012), « Les politiques d'investissement social à travers le monde », Document Cnaf.

Donzelot J. (1996), « Les transformations de l'intervention sociale face à l'exclusion ", in Paugam S. (dir.), L'exclusion : l'état des savoirs, Paris, La Découverte, p. 88-100.

Duflo E. (2003), "La politique économique à l'épreuve des faits », Finances \& Développement, septembre, p. 4-7.

Dufour P., Jenson J. (2003), « Des politiques d'emploi à la lutte contre la pauvreté des travailleurs : l'État d'investissement social dans les régimes libéraux de bien-être ", Working Paper $n^{\circ} 10$ of the project : Fostering Social Cohesion: A Comparison of New Policy Strategies.

Dworkin G. (1988), The Theory and Practice of Autonomy, Cambridge, Cambridge University Press.

Esping-Andersen G. (1990/trad. fr. 1999), Les trois mondes de l'État-providence : essai sur le capitalisme moderne, Paris, Puf.

Esping-Andersen G., Gallie D., Hemerrijck A., Myles J. (2002), Why We Need a New Welfare State, Oxford, Oxford University Press.

Esping-Andersen G., Palier B. (2008), Trois leçons sur l'État-providence, Paris, Éditions du Seuil.

Eyraud F., Marsden D., Sylvestre J.-J. (1990), « Marché professionnel et marché interne du travail en Grande-Bretagne et en France », Revue internationale du travail, vol. $129, \mathrm{n}^{\circ} 4$, p. 551-569.

Faure A., Pollet G., Warin P. (dir.) (1995), La construction du sens dans les politiques publiques : débats autour de la notion de référentiel, Paris, L'Harmattan.

Fiszbein A., Schady N.en collaboration avec Ferreira F.H.G., Grosh M., Keleher N., Olinto P., Skoufias E. (2009), Transferts monétaires conditionnels : réduire la pauvreté actuelle et future, Banque mondiale, Washington.

Fitoussi J.-P., Rosanvallon P. (1998), Le nouvel âge des inégalités, Paris, Éditions du Seuil.

Fougeyrollas A., Le Mouël P., Zagamé P. (2005), « Les nouvelles théories de la croissance en application. L'évaluation des politiques structurelles, le cas du protocole de Kyoto », Revue économique, vol. 56, n 5, p. 1089-1105. 
Fraser N. (2005), Qu'est-ce que la justice sociale ?, Paris, La Découverte.

Fraser N. (2010), « Marchandisation, protection sociale et émancipation. Les ambivalences du féminisme dans la crise du capitalisme ", Revue de l'OFCE, $\mathrm{n}^{\circ} 114$, p. $11-28$.

Gadrey J., Jany-Catrice F. (2012), Les nouveaux indicateurs de richesse, $3^{e}$ éd., Paris, La Découverte.

Gazier B., Palier B., Périvier H. (2014), Refonder le système de protection sociale. Pour une nouvelle génération de droits sociaux, Paris, Presses de Sciences Po.

Giddens A. (1990/trad. fr. 1994), Les conséquences de la modernité, Paris, L'Harmattan.

Guellec D., Ralle P. (2003), Les nouvelles théories de la croissance, Paris, La Découverte.

Guillemard A.-M. (dir.) (2008), Où va la protection sociale ?, Paris, Puf.

Hirsch M. (2011), Sécu : objectif monde. Le défi universel de la protection sociale, Paris, Stock.

ILO (2012), Social Protection Floors Recommendation, Recommandation ILO $\mathrm{n}^{\circ} 202$, 14 June.

Jacobs E., Pickard J., Parker G. (2011), « Big Society, grand fiasco ? », Financial Times, 16 février.

Jami I. (2009), « Considérer le problème plus que l'identité », Entretien avec Judith Butler, numéro spécial, "Pensées critiques », Mouvements, p. 117-130.

Jenson J. (2011), « Politiques publiques et Investissement social : quelles conséquences pour les femmes », Cahier du genre, hors-série, $n^{\circ} 2$, p. 21-43.

Jenson J., Lépinard É. (2009), « Penser le genre en science politique. Vers une typologie des usages du concept ", Revue française de science politique, vol. $59, n^{\circ} 2$, p. $183-201$.

Jobert B. (1993), "Représentations sociales, controverses et débats dans la conduite des politiques publiques ", Revue française de science politique, vol. $42, n^{\circ} 2$, p. 219-234.

Joly P. (1993), " Le ralentissement de la productivité, faits et causes ", in Guellec D. (dir.), Innovation et compétitivité, Collection Insee-Méthodes, $n^{\circ} 37 / 38$, novembre, p. 39-67.

Jones A. (2006), « L'apparition de la notion de participation dans la pensée et le discours du développement », REVEL, Revue électronique de l'UNS Sophia Antipolis.

Lafore R. (2004), « La décentralisation de l'action sociale, l'irrésistible ascension du "département providence" ", Revue française des affaires sociales, $\mathrm{n}^{\circ} 4$, p. 19-34.

Lemière S. (dir) (2013), L'accès à l'emploi des femmes : une question de politiques, Rapport remis à la ministre du Droits des femmes, décembre.

Letablier M.-T. (2007), « Comment l'Europe agit-elle sur la question des acteurs ? La famille et les politiques communautaires ", Informations sociales, $\mathrm{n}^{\circ} 139$, p. 128-139.

Lewis J. (1995), « Égalité, différence et rapports sociaux de sexe dans les Étatsprovidence du XX siècle ", in EPHESIA, La place des femmes. Les enjeux de l'identité et de l'égalité au regard des sciences sociales, Paris, La Découverte, p. 407-422. 
Lewis J. (1998), Gender, Social Care and Welfare State Restructuring in Europe, Aldersshots, Ashgate.

Mahieu F.-R. (2006), « L'anthropologie économique d'Amartya Sen », Horizon Local, Globenet, 2 avril.

Michaud E. (2007), « Les États, la Banque mondiale et le FMI face aux "stratégies de réduction de la pauvreté" ", in Lochak D. (dir.), Mutations de l'État et protection des droits de l'homme, Nanterre, Presses universitaires Paris Ouest, p. 237-250.

Molyneux M. (2007), « Change and Continuity in Social Protection in Latin America: Mothers at the Service of the State? ॥, Gender and Development, Programme Paper, $\mathrm{n}^{\circ}$ 1, United Nations Research Institute for Social Development, May.

Morel N. (2013), " L'État-providence suédois comme modèle social productif », Politiques sociales et familiales, $\mathrm{n}^{\circ} 112$, p. 39-49.

Muller P. (2005), « Esquisse d'une théorie du changement dans l'action publique : structures, acteurs, cadres cognitifs », Revue française de science politique, vol. $55, n^{\circ} 1$, p. 155-187.

de Munck J., Zimmermann B. (dir.) (2008), La liberté au prisme des capacités, Amartya Sen au-delà du libéralisme, Raisons Pratiques, $n^{\circ} 18$, Paris, Éditions de l'EHESS, p. 215-235.

Nicole-Drancourt C. (2001), " Histoire de la mise au travail des jeunes ", in Nicole-Drancourt C., Roulleau-Berger L., Les jeunes et le travail : 19502000, Paris, Puf.

Nicole-Drancourt C. (2007), « L'approche par les parcours et les cycles de vie, un rempart contre les discriminations ? ", Retraite et Société, $n^{\circ}$ 51, p. 175-205.

Nicole-Drancourt C. (2011), Donner du sens aux réformes. De l'équation sociale fordiste à la nouvelle équation sociale, l'enjeu des réformes dans l'ordre du genre, Habilitation à Diriger des Recherches, Paris, EHESS.

Nicole-Drancourt C. (2013), "Activation de la protection sociale : un droit à revenu universel de type nouveau ? », Mouvements, n 73, p. 124-132.

OCDE (1989), Perspectives de l'emploi de l'OCDE. Vers le plein emploi : l'ajustement structurel pour une société active, Paris.

OCDE (1995), Perspectives de l'emploi de l'OCDE. Améliorer l'efficacité des politiques actives du marché du travail, Paris.

OCDE (1996), Perspectives de l'emploi de l'OCDE. Contrecarrer les risques d'exclusion du marché du travail, Paris.

OCDE (1998a), L'investissement dans le capital humain - Une comparaison internationale, Paris.

OCDE (1998b), Perspectives de l'emploi de l'OCDE. Vers une politique sociale axée sur l'emploi, Paris.

OCDE (1999), L'économie mondiale de demain. Vers un essor durable ?, Paris.

OCDE (2001), Du bien-être des nations. Le rôle du capital humain et social, Paris.

OCDE (2014), Examens de l'OCDE sur la Gouvernance publique. Ensemble pour améliorer les services publics, Paris.

Orloff A.S. (1993), " Gender and the Social Rights of Citizenship: The Comparative Analysis of Gender Relations and Welfare States ", American Sociological Review, vol. 58, $n^{\circ} 3$, p. 303-328.

Palier B. (2005), « Vers un État d'investissement social. Pistes pour une redéfinition de la protection sociale ", Informations sociales, $n^{\circ} 128$, p. 118-128. 
Palier B. (2014), « La stratégie d'investissement social », Les études du Conseil économique, social et environnemental, février.

Pisani-Ferry J. (2000), Plein emploi, Rapport du Conseil d'analyse économique, Paris, La Documentation française.

Pnud (2014a), Pérenniser le progrès humain : réduire les vulnérabilités et renforcer la résilience, Rapport sur le développement humain.

Pnud (2014b), Autonomisation des jeunes pour un avenir durable. Stratégie du Pnud pour la jeunesse 2014-2017.

Robelin J. (dir.) (2004), « Habermas et la compréhension démocratique du droit international », in Robelin J. (dir.), Kant Anti-Kantien, Rouen, Publications Université Rouen.

Rosanvallon P. (1981), La crise de l'État-providence, Paris, Éditions du Seuil.

Rosanvallon P. (1995), La nouvelle question sociale. Repenser l'État-providence, Paris, Éditions du Seuil.

Rosanvallon P. (2006), La contre démocratie : la politique à l'âge de la défiance, Paris, Éditions du Seuil.

Sainsbury D. (ed.) (1994), Gendering Welfare States, London, Sage Publications.

Sen A. (1979), « Utilitarianism and Welfarism », The Journal of Philosophy, vol. 76, $n^{\circ}$ 9, September, p. 463-489.

Sen A. (1984), « Rights and Capabilities », in Sen A., Resources, Values and Development, Oxford, Blackwell/Cambridge, Harvard University Press.

Sen A. (1991), « La liberté individuelle : une responsabilité sociale », Esprit, mars.

Stiglitz J., Sen A., Fitoussi J.-P. (2009), Rapport de la Commission sur la mesure des performances économiques et du progrès social.

Revue Tiers Monde (2013), L'écriture de l'histoire dans les pays en développement, $n^{\circ} 216$.

Trenta A. (2014), La participation associative dans les quartiers populaires. Associations, problèmes publics et configurations politiques locales dans la périphérie urbaine de Paris et de Buenos Aires, Thèse de doctorat en sociologie, Conservatoire national des arts et métiers.

Vielle P. (2002), Les femmes et le droit, Presses des Facultés universitaires de Saint Louis.

Vielle P., Pochet P., Cassiers I. (dir.) (2005), L'Etat social actif, vers un changement de paradigme?, Bruxelles, Peter Lang.

Viriot Durandal J.-P. (2004), « Travailleurs âgés et politiques publiques : une perspective comparée », Congrès AFS, 25 février.

Wim K. (2003), L'emploi, l'emploi et l'emploi: créer plus d'emploi en Europe, Rapport du Groupe d'analyse pour la Stratégie de Lisbonne. 